\title{
Use of Radio Occultation to Evaluate Atmospheric Temperature Data from Spaceborne Infrared Sensors
}

\author{
Thomas P. Yunck ${ }^{1,}$, Eric J. Fetzer ${ }^{2}$, Anthony M. Mannucci ${ }^{2}$, Chi O. Ao $^{2}$, F. William Irion ${ }^{2}$, \\ Brian D. Wilson ${ }^{2}$, and Gerald John M. Manipon ${ }^{3}$ \\ ${ }^{1}$ GeoOptics LLC, Pasadena, CA 91103, USA \\ ${ }^{2}$ Jet Propulsion Laboratory, California Institute of Technology, Pasadena, CA 91109, USA \\ ${ }^{3}$ Raytheon DSIO, Pasadena, CA 91101, USA
}

Received 27 June 2007, accepted 8 December 2007

\begin{abstract}
With its high accuracy, stability, and worldwide coverage GPS radio occultation offers an attractive means of independently validating and calibrating the world's premier weather and climate sensors. These include such instruments as AIRS, AMSU, and MODIS on NASA's EOS platforms, and similar systems on operational weather satellites. GPSRO also offers a valuable comparison standard for global weather analyses, such as those produced by NOAA's National Center for Environmental Predictions (NCEP) and the European Centre for Medium-Range Weather Forecasts (ECMWF).

We have studied the performance of GPSRO temperature profiles through comparisons of coincident data from CHAMP and SAC-C, as well as from COSMIC. We have also compared GPSRO temperature profiles with nearby profiles from AIRS (Atmospheric Infrared Sounder), carried on NASA's Aqua platform, and with the ECMWF analyses. Our principal findings are:

- AIRS and ECMWF temperature profiles depart in systematic ways from GPSRO profiles. These departures are highly repeatable and vary by geographical region.

- There is significant correlation between the AIRS and ECMWF departures from GPSRO, not explainable by GPSRO error. This may arise because AIRS retrievals are initialized with estimates derived from ECMWF training samples.

- ECMWF single-profile RMS temperature deviations range between 0.6 and $1.8 \mathrm{~K}$ and are at a maximum near the tropopause. Biases are typically below $0.5 \mathrm{~K}$.

- AIRS single-profile RMS temperature deviations range between 0.9 and $2.2 \mathrm{~K}$ and are also at a maximum near the tropopause. Biases are typically below $0.5 \mathrm{~K}$ but reach $1 \mathrm{~K}$ near the tropopause in the Antarctic.
\end{abstract}

Key words: AIRS, GPS, Comparisons, Temperature profiles, Temperature biases

Citation: Yunck, T. P., E. J. Fetzer, A. M. Mannucci, C. O. Ao, F. W. Irion, B. D. Wilson, and G. J. M. Manipon, 2009: Use of radio occultation to evaluate atmospheric temperature data from spaceborne infrared sensors. Terr. Atmos. Ocean. Sci., 20, 71-85, doi: 10.3319/TAO.2007.12.08.01(F3C)

\section{INTRODUCTION}

Atmospheric sounding by GPS Radio Occultation (GPSRO) is coming of age. The Central Weather Bureau in Taiwan now uses COSMIC data in operational forecasts, as do NCEP in the US and ECMWF in Europe. The precision and stability of GPSRO can enhance the observational basis for studying long-term climate change and make GPSRO well suited for another critical function: assessing and calibrating the performance of conventional atmospheric sen-

\footnotetext{
* Corresponding author

E-mail:geooptics@charter.net
}

sors. These include the workhorse sounders on NASA's research missions, such as Terra and Aqua, and on operational weather satellites.

One of these flagship sensors is the Atmospheric Infrared Sounder (AIRS), a high spectral resolution IR radiometer on Aqua. As a featured component of NASA's Earth Observing System (EOS) fleet of remote sensing instruments, AIRS provides data for studying a range of atmospheric and surface processes and their roles in Earth's climate. To help assess the value of AIRS (and of high spectral resolution IR sounding generally) analysts must characterize 
and quantify its range of performance. In this study we apply GPS temperature profiles to illuminate properties of AIRS temperature retrievals that are otherwise difficult to observe.

\section{GPSRO AS A VALIDATION STANDARD}

Several unique qualities make GPSRO (illustrated in Fig. 1) suited to the roles of calibration and verification. It works by physical principles entirely different from radiometry to provide an independent comparison. Over its most effective altitude range (roughly $5-25 \mathrm{~km}$ ) GPSRO exhibits an absolute bias of $<0.1 \mathrm{~K}$, single-profile repeatability of $<1 \mathrm{~K}$, and vertical resolution of $100-1000 \mathrm{~m}$ in temperature retrievals (e.g., Ho et al. 2009). (GPS precision is discussed further in the section, Basis of GPSRO Performance Claims). Uniquely among spaceborne sensors, GPSRO provides the absolute geopotential heights of all its measurements, with an accuracy of better than $10 \mathrm{~m}$ (Leroy 1997). GPSRO profiles are collected almost uniformly around the globe, including over oceans where AIRS performance is most dependable, and performance is essentially unaffected by clouds, dust, and weather, or by the nature of the surface below.

\section{AIRS OVERVIEW}

With 2378 IR channels, AIRS is among the most advanced atmospheric sensors in operation today. Space News has noted, "The most detailed atmospheric soundings currently come from the AIRS instrument on NASA's Aqua satellite" (18 September 2006). In discussing AIRS it is helpful to distinguish two modes of use: as a pure radiometer, producing radiance measurements at multiple frequencies for assimilation into forecast models; and as a means for directly retrieving geophysical products, such as profiles of temperature, moisture, and ozone, independent of assimilation models.

Assimilation of AIRS radiances has been shown to produce consistent and significant improvement in weather forecasts (e.g., Chahine et al. 2006; Healy et al. 2006). We note, however, that relatively few channels are needed to produce this improvement. ECMWF, for example, ingests 60 of the 2378 radiances operationally.

The complexity of AIRS derives largely from its goal of producing direct geophysical retrievals of high accuracy and vertical resolution. The information provided by hundreds of channels may permit the extraction of such products unmediated by assimilation models. The specification for AIRS temperature retrievals, which dates back more than 25 years, is 1 Kelvin absolute accuracy at $1 \mathrm{~km}$ vertical resolution from the boundary layer to the upper stratosphere. A good deal of effort has gone into testing that claim, primarily by comparison with other techniques. Chahine et al. (2006), for example, claim a cloud clearing error of $0.6 \mathrm{~K}$ and a collocation error (with radiosondes) of $0.8 \mathrm{~K}$.

Divakarla et al. (2006) compared AIRS temperature and moisture profiles with near-coincident radiosondes, or RAOBs. Figure 2 gives a typical result showing the RMS differences between RAOB measurements and AIRS for the less common "clear-only" conditions (dashed lines) and the more common "cloud-cleared" measurements in which microwave data from AMSU aid the IR retrieval under partial cloud conditions. The curves in Fig. 2 are for the full globe, including measurements over oceans and land. We see RMS temperature differences of $0.9-1.6 \mathrm{~K}$ up to $100 \mathrm{hPa}$ for clear-only profiles, and $1.2-1.7 \mathrm{~K}$ for cloud-cleared profiles.

The AIRS validation team at JPL performs regular comparisons of AIRS retrievals between $\pm 50^{\circ}$ latitude with ECMWF global analyses on so-called AIRS "focus days." Figure 3 shows the AIRS-ECMWF RMS temperature deviations (after removal of the mean offset) for retrievals over oceans and land for 14 focus days between September 2002 and December 2004. The comparisons are restricted to "Quality 0" AIRS data, which is the best of five quality levels reported by AIRS. Over water we see RMS deviations of $0.7-1.3 \mathrm{~K}$ for a wide altitude range with the deviations over land somewhat higher.

These results would appear to support the claim of $1 \mathrm{~K}$ AIRS temperature accuracy when we consider that the observed deviations include the effects of both measurements in each comparison. If the measurements are fully inde-

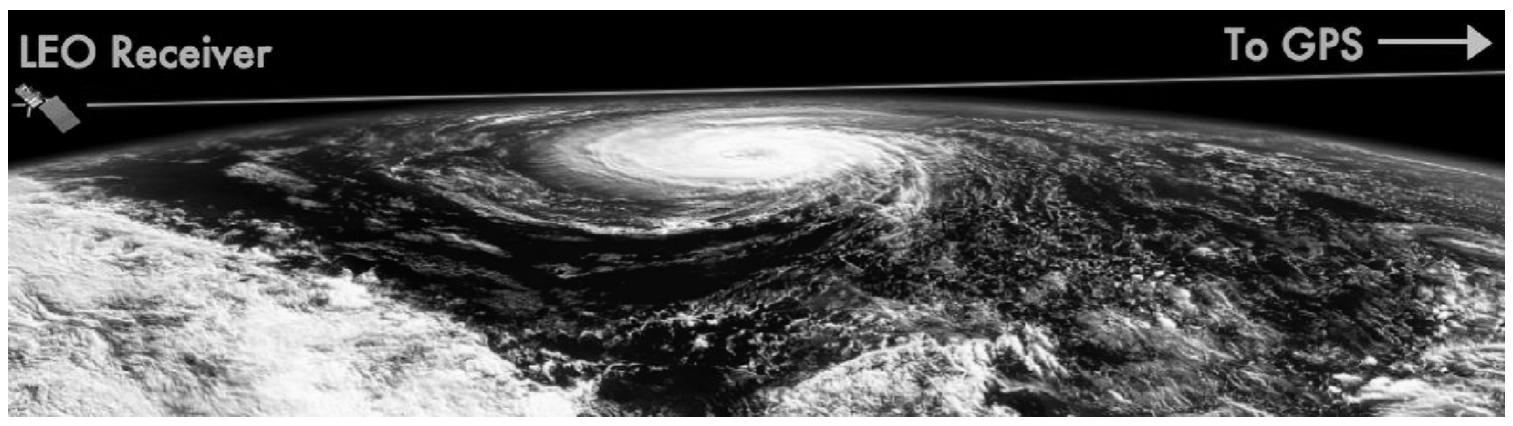

Fig. 1. Illustration of the GPS limb-sounding geometry. 
pendent (an assumption we shall challenge below) and roughly comparable in their errors, it follows that the "Quality 0" AIRS single-profile sigma is at or below $1 \mathrm{~K}$ from the surface to $30 \mathrm{~km}$ altitude. We note, however, that the ECMWF analyses depend strongly on RAOB inputs and (as we shall argue) the AIRS retrievals are partially influenced by the ECMWF model, giving rise to the possibility of correlations that could lead to mutual agreement surpassing actual accuracy (see also Divakarla et al. 2006). Thus these comparisons, while encouraging, are inconclusive and we remain uncertain whether AIRS performance might actually be better or worse than indicated in Figs. 2 and 3.

The high precision and low bias of GPSRO temperatures over its effective altitude range allows us to examine that performance more closely. The "effective altitude range" varies geographically and is determined at the low end by the concentration of moisture in the atmosphere, and at the high end by the GPS signal-to-noise ratio, which limits how high we can observe in the rapidly thinning atmosphere. The current practical upper limit is about $30 \mathrm{~km}$, though performance is best to about $25 \mathrm{~km}$. Since the atmospheric refractivity depends on both temperature and moisture content and GPSRO alone can't readily distinguish between the two, we must limit the lower range to altitudes where the moisture effect is insignificant; in polar regions,

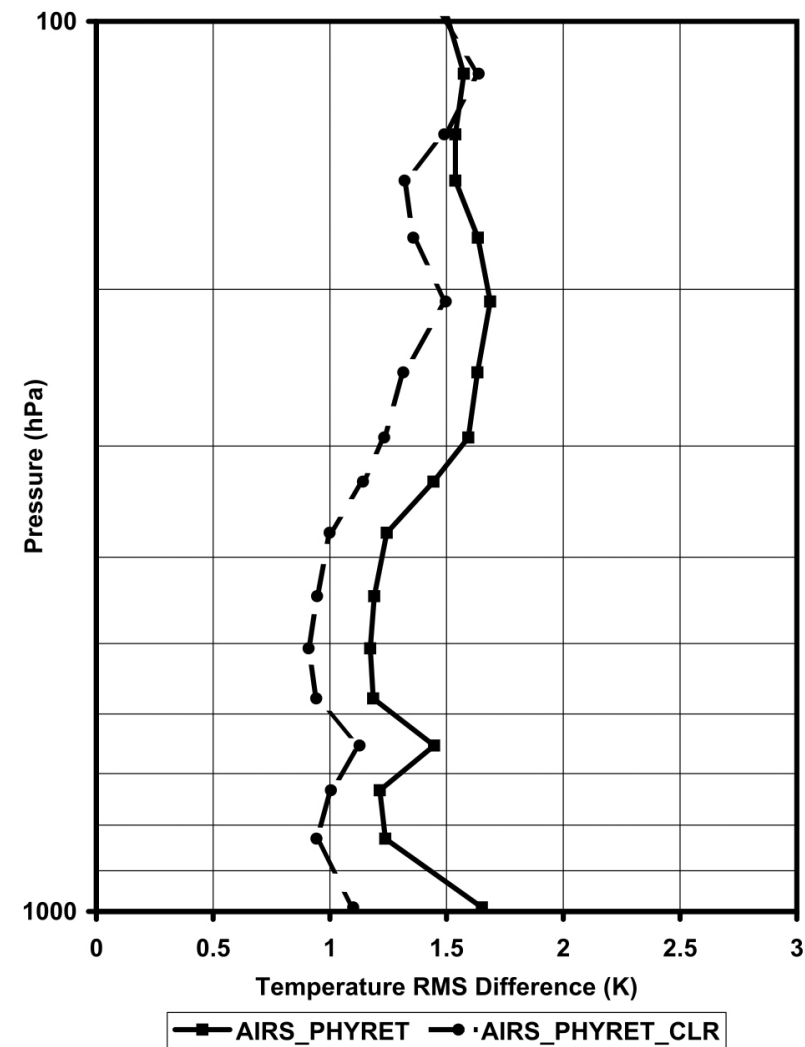

Fig. 2. AIRS-RAOB temperature RMS difference (from Divakarla et al. 2006) this is typically $2-3 \mathrm{~km}$, at mid latitudes about $5 \mathrm{~km}$, and in the tropics $8-10 \mathrm{~km}$.

We have performed a series of comparisons involving GPSRO, AIRS, and ECMWF temperature profiles from 2003 over the effective GPS altitude range. As 2003 predates COSMIC, the GPS data were taken from the CHAMP and SAC-C missions. For the comparisons we must identify AIRS and GPS measurements that are nearly coincident in space and time. For that purpose we have defined a coincident pair as a pair of profiles occurring within $200 \mathrm{~km}$ and 2 hours of one another.

\section{COMPARISON OF AIRS, GPS AND ECMWF: $30-60^{\circ} \mathrm{N}$}

For the initial comparisons we selected a region where all three methods are expected to work well, which meant avoiding the poles, where ice and snow can confound AIRS

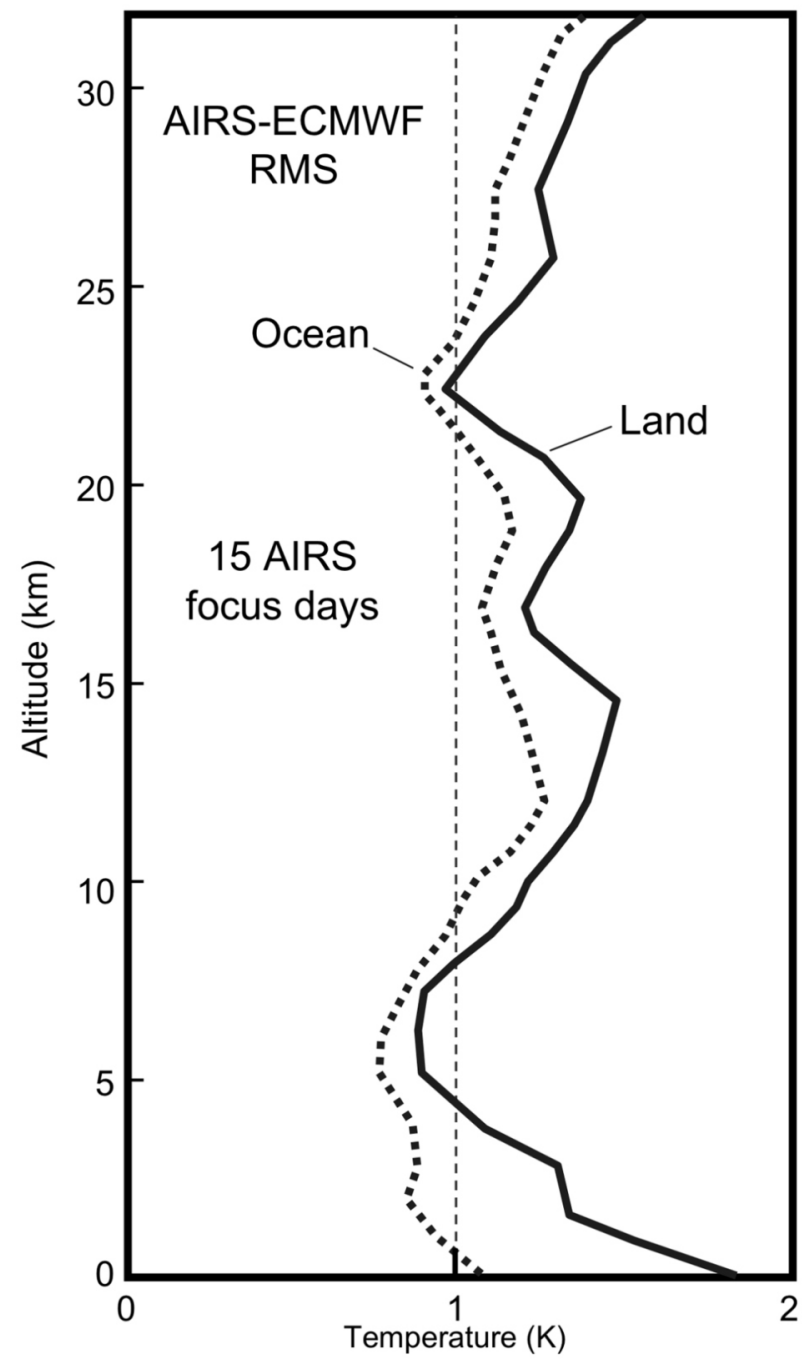

Fig. 3. AIRS-ECMWF RMS temperature deviations for 15 AIRS focus days from September 2002 to December 2004 (adapted from Fetzer et al. 2005). 
cloud detection, and selecting regions with dense coverage by radiosondes, a critical input for ECMWF. We chose the latitude band $30-60^{\circ} \mathrm{N}$, ("Mid North") which spans much of North America, Europe, and Asia and has exceptional radiosonde coverage. Within that region we identified all AIRSGPS coincident pairs for 2003, then retrieved the ECMWF values for those locations and times. (Tests showed that comparison statistics were unaffected by whether the ECMWF profile was computed for the precise AIRS location, the GPS location, or in between.) All profiles were smoothed to a $2 \mathrm{~km}$ vertical resolution to ensure, to the degree possible, that like quantities were being compared. That value was determined largely from the apparent vertical resolution available from AIRS. For the three pair-wise match-ups - AIRS-ECMWF, AIRS-GPS, and GPS-ECMWF - we computed the temperature difference at $2 \mathrm{~km}$ vertical intervals and computed the mean offsets and the RMS deviations about the mean offsets for all of 2003.

\subsection{A Conundrum}

Figure 4 shows the RMS temperature deviations (RMS scatter about the mean offsets) for each of the three comparison pairs for 765 match-ups occurring in the Mid North in 2003. What stands out is the larger deviation for the GPS-AIRS match-up than for the other two, and that, for the most part, the two match-ups involving GPS have the largest

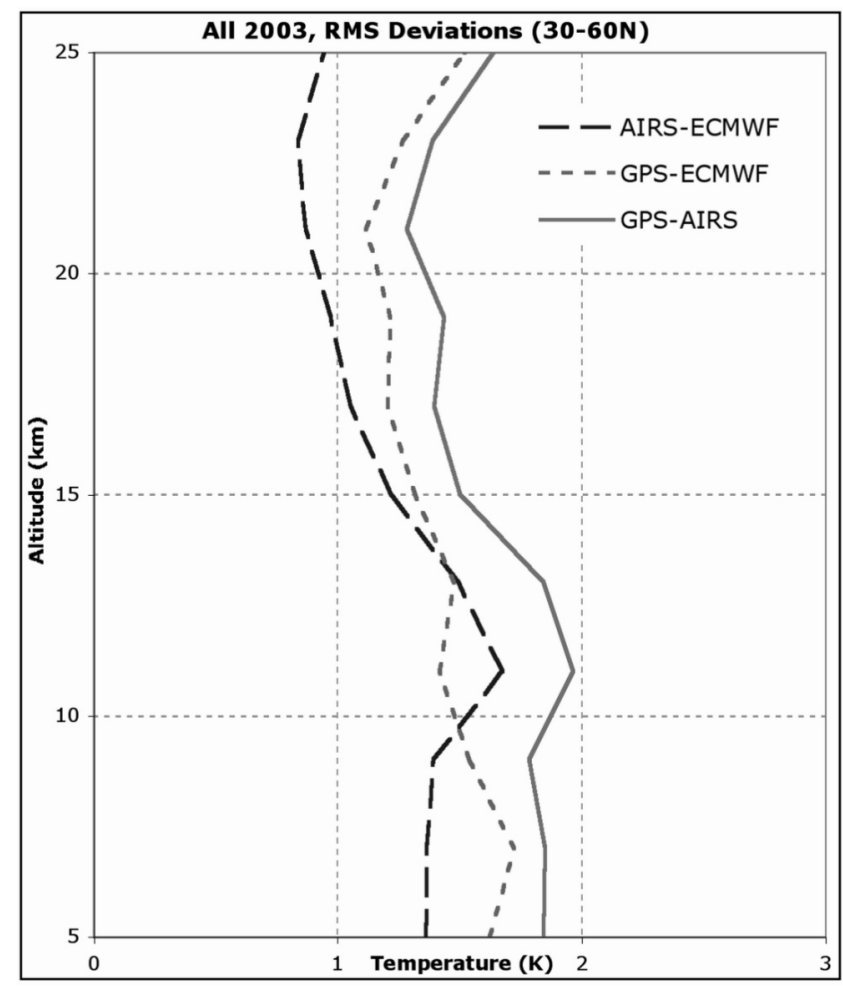

Fig. 4. Mid North RMS deviations about the mean differences for 765 three-way match-ups. deviations. This is puzzling since GPSRO precision is believed to be well under $1 \mathrm{~K}$ over most of this range (e.g., Kursinski et al. 1997; Rocken et al. 1997; Hajj et al. 2004; Kuo et al. 2004) and to surpass the precision of both AIRS and ECMWF, at least below $20 \mathrm{~km}$. If that were true, and if the three datasets were mutually independent, then the AIRS-ECMWF pair would show the greatest RMS scatter. Instead, it shows the least.

There are two plausible explanations. Either the GPS measurement precision is far worse than the evidence to date has indicated, or there is a sizable correlation between AIRS and ECMWF to account for their better-than-expected agreement. Here we will examine these possibilities more closely.

\subsection{Basis of GPSRO Performance Claims}

Interpretation of these results depends strongly on our understanding of the performance of GPSRO. There are several bases for that understanding. First, many investigators have performed theoretical and simulation studies of GPSRO over the past fifteen years. The most comprehensive is Kursinski et al. (1997), who examined all known major error sources. Figure 5, adapted from that study, shows the estimated total error for a single profile in the tropics and at mid and high latitudes for altitudes from 5 to $30 \mathrm{~km}$.

Perhaps more revealing are direct comparisons of near-coincident GPSRO profiles from separate instruments. We now have hundreds of such coincident pairs from

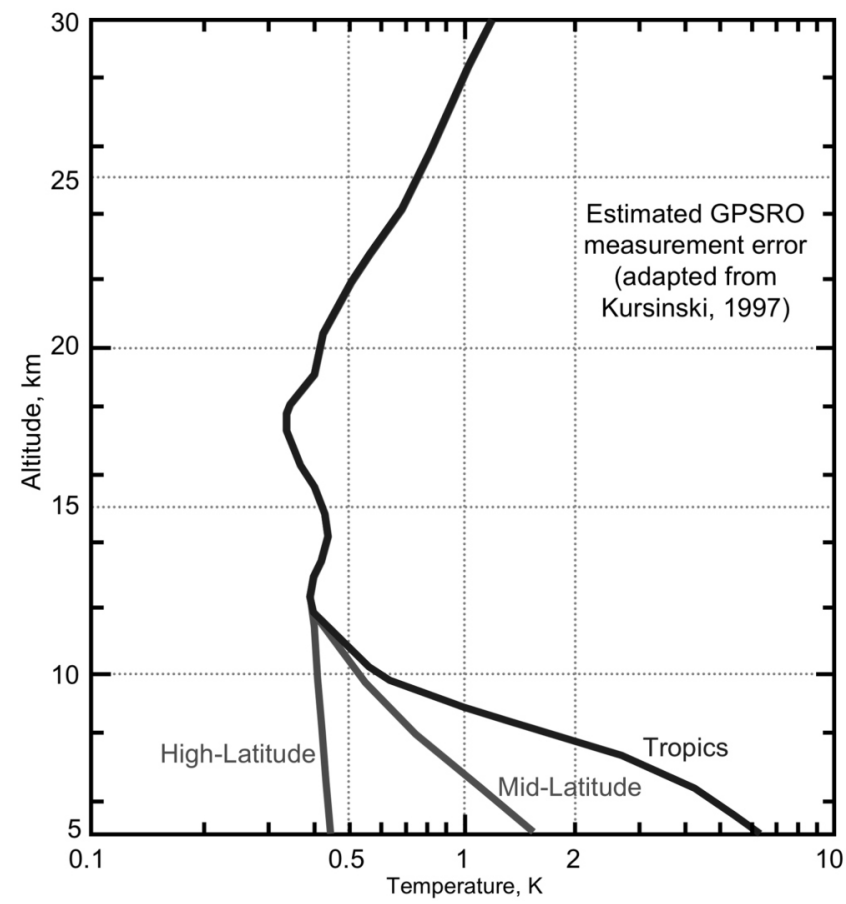

Fig. 5. Expected GPSRO temperature error in three regions (adapted from Kursinski et al. 1997). 
CHAMP and SAC-C and, more recently, several thousand from COSMIC. As the CHAMP/SAC-C coincidences are typically up to $2 \mathrm{hrs}$ and $200 \mathrm{~km}$ apart, there are real differences between the quantities being measured. These differences were estimated in earlier CHAMP/SAC-C studies (e.g., Hajj et al. 2004) to be $0.1-0.4 \mathrm{~K}$, depending on separation distance and time, as well as height. COSMIC has now produced thousands of pairs within 1 minute and $10 \mathrm{~km}$ of one another; for those, the real temperature differences can be considered negligible. The COSMIC studies have more clearly mapped the effect of separation distance on temperature agreement and confirmed the $0.1-0.4 \mathrm{~K}$ estimate for real temperature differences for separations up to $200 \mathrm{~km}$ in the upper troposphere and lower stratosphere (e.g., Anthes 2006, p. 21).

Figure 6 shows the estimated single-profile RMS deviations derived from comparisons of retrievals from CHAMP and SAC-C, COSMIC 1 and 3, and COSMIC 2 and 3. The actual RMS deviation curves have been smoothed slightly and then divided by the square root of 2 to give the single-profile estimates. While the curves are similar the COS-

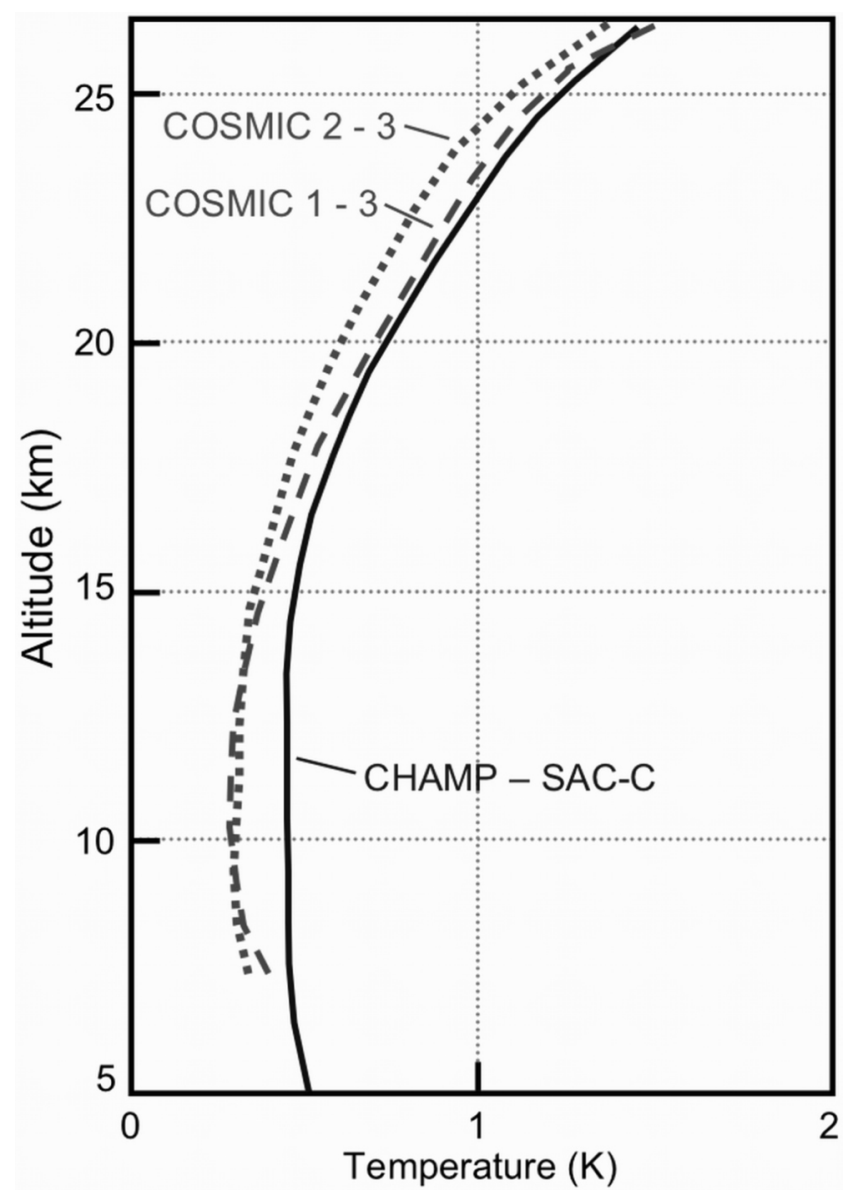

Fig. 6. Plots of estimated single-profile GPS temperature deviations derived from coincident GPS-GPS matchups from three satellite pairs: CHAMP/SAC-C, COSMIC 1 and 3, and COSMIC 2 and 3. The COSMIC match-ups were much closer in space and time.
MIC performance is somewhat better than for the earlier missions. At higher altitudes where performance is limited by signal-to-noise ratio (SNR), this is likely due to the higher gain of the COSMIC occultation antennas. Below $15 \mathrm{~km}$, the improvement may result from the closer proximity of the COSMIC coincident profiles.

These comparisons do not reveal measurement accuracy, only precision or repeatability. There can be systematic errors, particularly below $8 \mathrm{~km}$ where water vapor becomes prominent, common to the coincident pairs that cancel in the differences. To model the GPSRO error for these studies we take a somewhat cautious approach. We use the CHAMP SAC-C curve from Fig. 6 to represent the random component and add in (in the root-sum-square, or RSS, sense) a systematic component based on the Kursinski et al. (1997) analysis. The result for the Mid North band is shown in Fig. 7. If anything, this may overstate the GPS error for this study since it is derived in part from GPS-GPS comparisons at $1 \mathrm{~km}$ vertical resolution; for the AIRS comparisons we have smoothed to $2 \mathrm{~km}$, which reduces the random component.

Let us assume for the moment that this accurately models the GPSRO temperature error and that GPSRO errors are independent of the errors in both AIRS and ECMWF. We can then estimate the single-profile deviations for both AIRS and ECMWF by performing an RSS (or quadrature) subtraction of the modeled GPSRO error (Fig. 7) from the observed GPS-AIRS and GPS-ECMWF deviation curves (Fig. 4). The results are shown as the two "Derived"

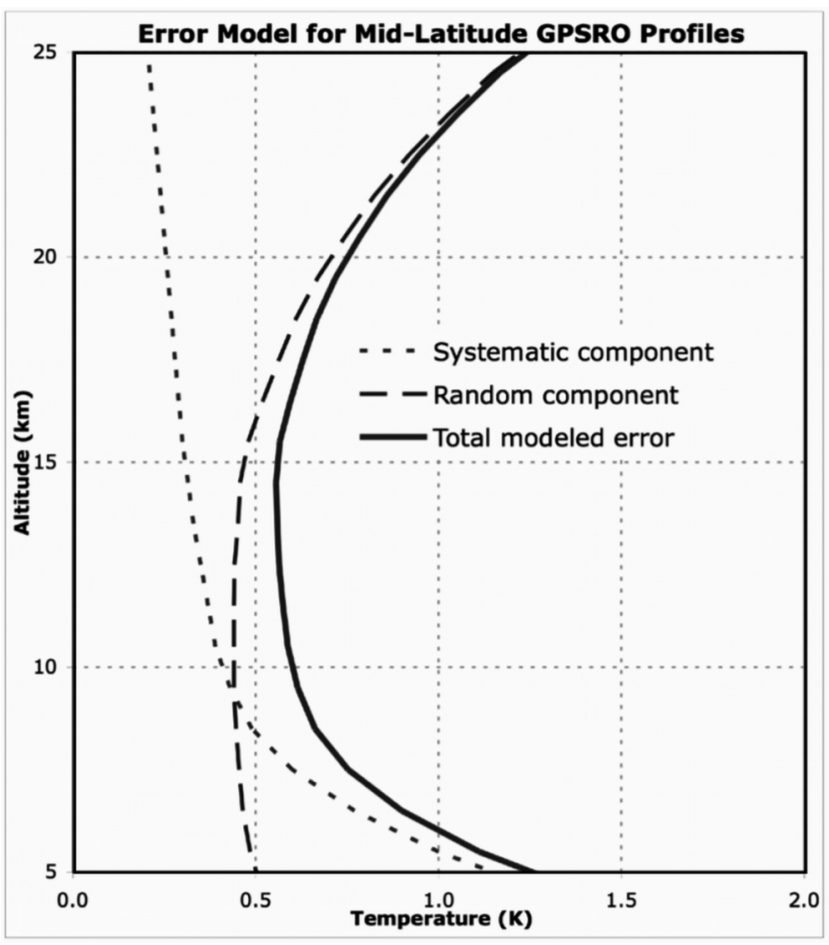

Fig. 7. Error model for individual mid-latitude GPSRO temperature profiles. 
deviations in Fig. 8. If the AIRS and ECMWF profiles were mutually independent, we would expect the RMS deviation for the AIRS-ECMWF comparison to be the RSS of the individual derived deviations for AIRS and ECMWF. This is also shown in Fig. 8 (rightmost dotted line) along with the AIRS-ECMWF deviation actually observed (dashed line in center) and the modeled GPS deviation.

The discrepancy is striking. Not only is the observed AIRS-ECMWF deviation far smaller than expected, it is smaller than the derived single-profile AIRS deviation and close to the single-profile ECMWF deviation. If our GPS model error is indeed accurate (a subject we address further below), then there must be a correlation between AIRS and ECMWF leading to error cancellation in the AIRS-ECMWF difference.

We can quantify this in terms of the required covariance of AIRS and ECMWF profiles with the formula,

$$
\begin{aligned}
\operatorname{Var}(A-E)= & \operatorname{Var}(A)+\operatorname{Var}(E)-2 \operatorname{Cov}(A, E) \\
& +\operatorname{Var}\left(A_{\text {true }}-E_{\text {true }}\right)
\end{aligned}
$$

where $\operatorname{Var}(\mathrm{A}$ - E) is the variance of the observed AIRSECMWF differences, $\operatorname{Var}(\mathrm{A})$ and $\operatorname{Var}(\mathrm{E})$ are the inherent variances for AIRS retrievals and ECMWF model values, and $\operatorname{Var}\left(\mathrm{A}_{\text {true }}-\mathrm{E}_{\text {true }}\right)$ is the variance of the true temperature differences at the observation points. Because the final term is generally small $(0.01-0.16)$ we can ignore it in the

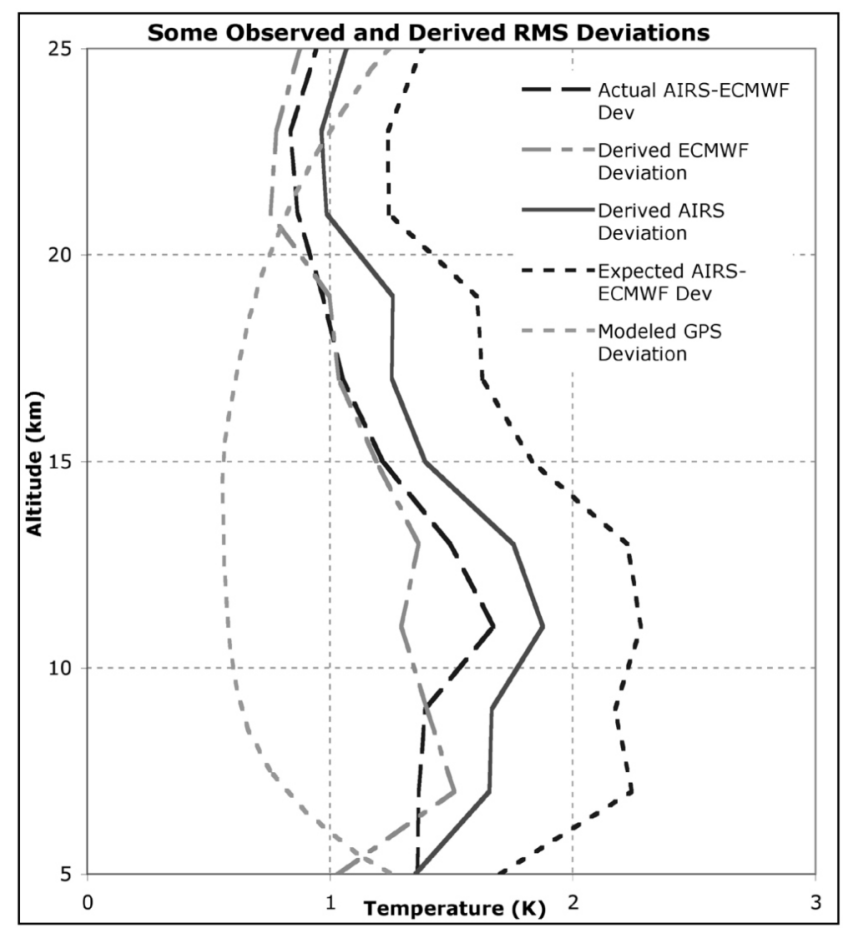

Fig. 8. Plots of the modeled GPS temperature deviation; the estimated AIRS and ECMWF deviations and expected AIRS-ECMWF joint deviation based on the GPS model error, assuming GPS is independent of AIRS and ECMWF; and the observed AIRS-ECMWF deviation. analysis. We can write this equation for each of the three pairings. Given the GPSRO error model in Fig. 7 and assuming GPSRO is uncorrelated with both AIRS and ECMWF, we can solve explicitly for the variances and covariance of AIRS and ECMWF profiles.

Figure 9 shows the resulting "covariation" (square root of the covariance) of AIRS and ECMWF temperature profiles (long dashed line) alongside the estimated deviations for the techniques individually, for the Mid North band (rightmost lines). Also shown for reference is the modeled GPS deviation and the correlation coefficient for AIRS and ECMWF, which varies between about 0.4 and 0.7 . We see that if the modeled GPS deviation is accurate, there is a sizable correlation between AIRS and ECMWF profiles and that the overall variance for AIRS is consistently greater than for ECMWF.

\subsection{Alternative Interpretation}

This interpretation depends fundamentally on our model for the single-profile GPSRO standard deviation (Fig. 7). The uncorrelated component of that error model is well constrained by coincident GPSRO comparisons, but the correlated component (i.e., the systematic component in Fig. 7), though based on both theory and observation, is less well determined. We can free up that component and trade off some hypothetical GPS systematic error against AIRS-

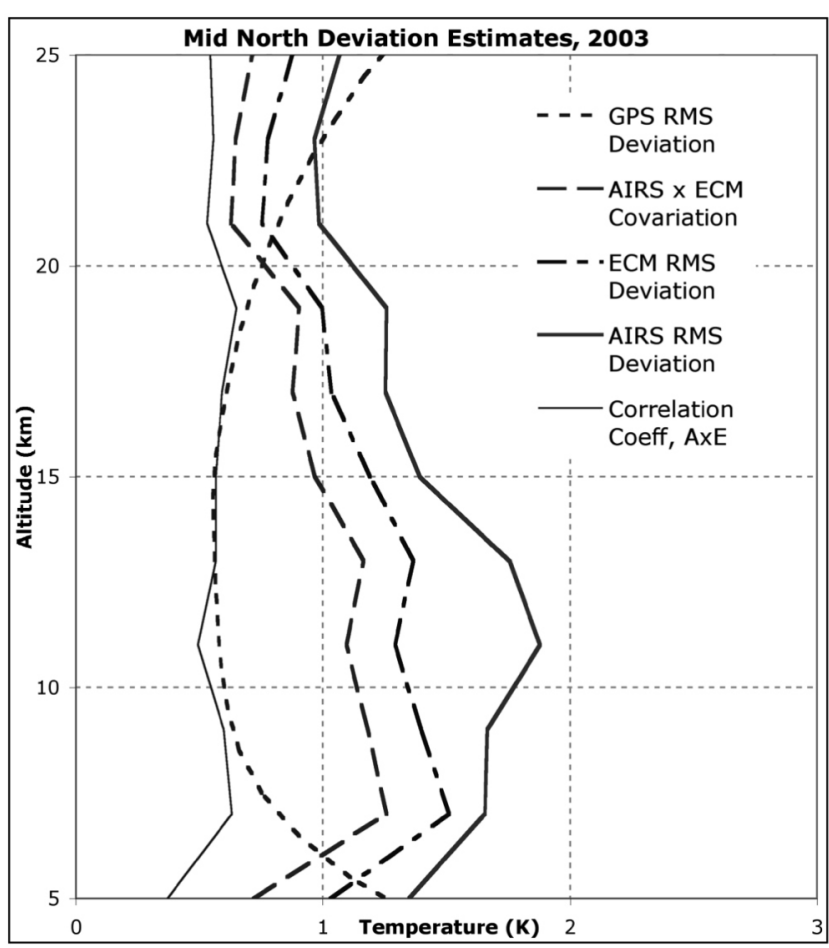

Fig. 9. Estimated AIRS and ECMWF RMS temperature deviations and the AIRS-ECMWF covariation, assuming the modeled GPS deviation. Also shown is the resulting AIRS-ECMWF correlation coefficient. 
ECMWF correlation: Increasing one decreases the other in a precisely known way. In the limit, if we set the AIRSECMWF correlation to zero, attributing all "excess" deviation to added GPS systematic error, we obtain the deviations shown in Fig. 10. This presents a markedly different picture, though one with obvious problems. The total GPS RMS deviation is above $1 \mathrm{~K}$ for the entire range, with nearly all of it (at least below $20 \mathrm{~km}$ ) due to common GPS variations that reliably disappear in the GPS-GPS differences. Many years of analysis have revealed no evidence or plausible mechanism for such large systematic GPSRO errors. Moreover, the assumption of zero AIRS-ECMWF correlation forces an improbably low RMS deviation for the ECMWF analysis, with values ranging between 0.4 and $0.8 \mathrm{~K}$. Global ECMWF analysis error is generally believed to be about twice that.

The GPSRO model error of Fig. 7 represents our best current information on GPSRO performance, and in fact is believed to somewhat overstate the errors. If that is true then we are confronted with an evident correlation between AIRS and ECMWF temperature profiles, which on the surface may seem to have no clear connection. Before presenting further evidence of such a correlation we describe a mechanism by which it might arise.

\subsection{The AIRS Retrieval Process}

To obtain a temperature profile from AIRS radiances the

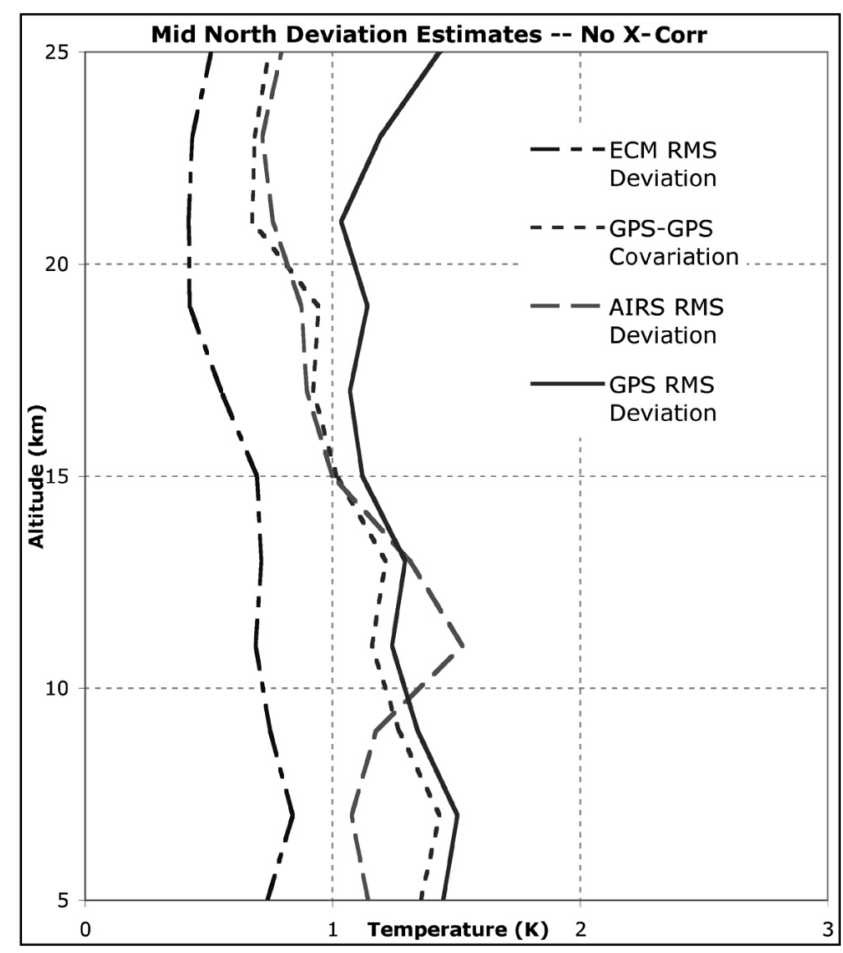

Fig. 10. Estimated individual RMS deviations assuming no correlation between AIRS and ECMWF profiles. The required GPS systematic error is shown by the dotted line. retrieval system first takes a subset of the 2378 radiances collected for each footprint, typically 200 , and multiplies them by a matrix of coefficients that map radiances to temperatures. This is called the "regression" step. These estimates then become the initial values in a complex estimation process employing the same set of radiances (Divakarla et al. 2006). Exactly the same regression coefficients are used to initialize all retrievals worldwide, every day, year-in and year-out. Where did these critical coefficients come from?

The regression coefficients were derived in a one-time training process using as its truth model the ECMWF global analysis for two days in August 2002. The AIRS regression coefficients are thus trained to map particular combinations of radiances characteristic of distinct atmospheric states into those states, not necessarily as they were, but as they were represented in the ECMWF analysis. To some degree, then, the ECMWF representations of different atmospheric states, and thus their inherent biases, are encoded in the AIRS regression coefficients.

On a given day a great variety of atmospheric states, occur around the globe. While those states change, the particular state in a given AIRS footprint will likely resemble, possibly quite closely, states occurring in the training period in 2002. Assuming similar atmospheric states give rise to similar radiance patterns (a basic tenet of radiometry), systematic misrepresentations of those states in the ECMWF model may be reproduced in the AIRS regression step. To what extent such systematic errors may persist over months or years is unknown, but model biases deriving from such factors as smoothing and the uneven distribution of RAOB inputs may well be similar in 2002 and 2003.

These heuristic arguments deal only with the initial AIRS regression step. A complex estimation process then transforms the regression output into the final AIRS temperature profiles. This raises the question of how much freedom is given in the estimation step to modify the regression results. If the solution is tightly constrained, final estimated values close to the a priori will result. Tight constraints are required when the information in the input data is weak and unable to carry the burden of the estimate. The question then becomes, "How tightly constrained are the AIRS temperature estimates?" Surprisingly little is known about this, though further work is underway by the AIRS team.

\section{SUPPORTING ANALYSIS I: THE TROPICAL TROPOSPHERE}

We can gain some insight into systematic errors by examining other regions where a particular technique is known to behave in a particular way. The tropics, for example, often exhibit a sharp tropopause, which can vary in altitude by several kilometers over time and space. Vertical smoothing inherent in the modeling process makes it impossible for the 
analyses to capture such features precisely, while the high vertical resolution of GPSRO can do so easily. Figure 11 shows an example in which nearly coincident GPSRO profiles from CHAMP and SAC-C pass through a sharp tropopause. Also shown are both the ECMWF and NCEP model profiles computed for the locations of the GPS profiles. These reveal characteristic model errors near a sharp tropopause: a smoothing down of the peak to produce a warm bias at the tropopause, along with a corresponding cold bias immediately above. This is typical of the distortion imposed on a sharp pulse that has gone through a low-pass (or smoothing) filter.

The sharpness of the cold peak can cause high variability in the model error around the tropopause. If the tropopause height moves up 1 or $2 \mathrm{~km}$, the cold bias previously above will be replaced by a warm bias. Since tropopause height varies by $1-2 \mathrm{~km}$ around the tropics, we see higher variation in the GPS-ECMWF differences in the tropopause region than elsewhere, a property entirely attributable to limitations of the model. (Note: Fig. 11 depicts an unusual tropopause outside the tropics; the tropical tropopause height tends to occur higher, at $15-18 \mathrm{~km}$.)

This characteristic of the tropical ECMWF model is evident in Fig. 12, which shows the RMS deviations for thousands of GPS-ECMWF comparisons (not just where there are AIRS matchups) for all of 2003, in three regimes: $30^{\circ}-90^{\circ}$ north; $30^{\circ}-90^{\circ}$ north and south combined; and the tropics, here defined as $\pm 30^{\circ}$. As no AIRS data were included, the comparisons in Fig. 12 were done at a $1 \mathrm{~km}$ vertical resolution rather than $2 \mathrm{~km}$. The contrast is dra-

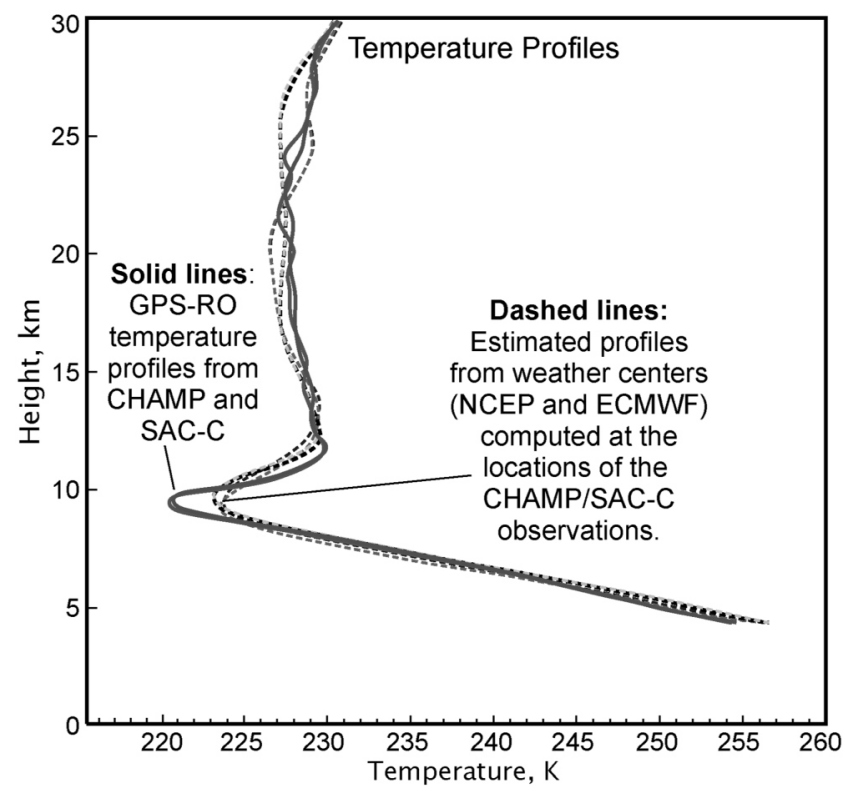

Fig. 11. A sharp high-latitude tropopause observed by CHAMP and SAC-C, along with the corresponding model analyses from ECMWF and NCEP, showing characteristic distortion by the analyses, which lack the resolution to capture the sharp feature. matic. In the tropics we see the expected high variation due to tropopause mis-modeling at $15-20 \mathrm{~km}$, with lower variation immediately below, increasing again as GPS descends into the tropical moisture. Outside the tropics we see an analogous but far less pronounced variation bulge at $9-13 \mathrm{~km}$, due, we believe, to the lower and generally less distinct non-tropical tropopause.

Returning to our match-ups, Fig. 13a shows the estimated individual RMS deviations for AIRS and ECMWF along with the estimated AIRS-ECMWF covariation from more than 2200 three-way pairings in the Tropics, where the GPSRO deviation has been modeled by the curve shown. In this case we have increased the GPS model variation below $8 \mathrm{~km}$ to account for significantly increased moisture in the tropics. Figure 13b shows the three estimated RMS deviations under the assumption of zero correlation between AIRS and ECMWF, allowing the systematic GPS error to grow.

The first plot shows the expected high deviation in the ECMWF model around the tropical tropopause. AIRS shows a similar pattern, though with considerably higher overall deviation, and the AIRS-ECMWF covariation is at nearly the full level of the ECMWF deviation. By contrast, the second scenario pushes the full deviation increase at the tropopause onto the GPS side of the ledger, though this con-

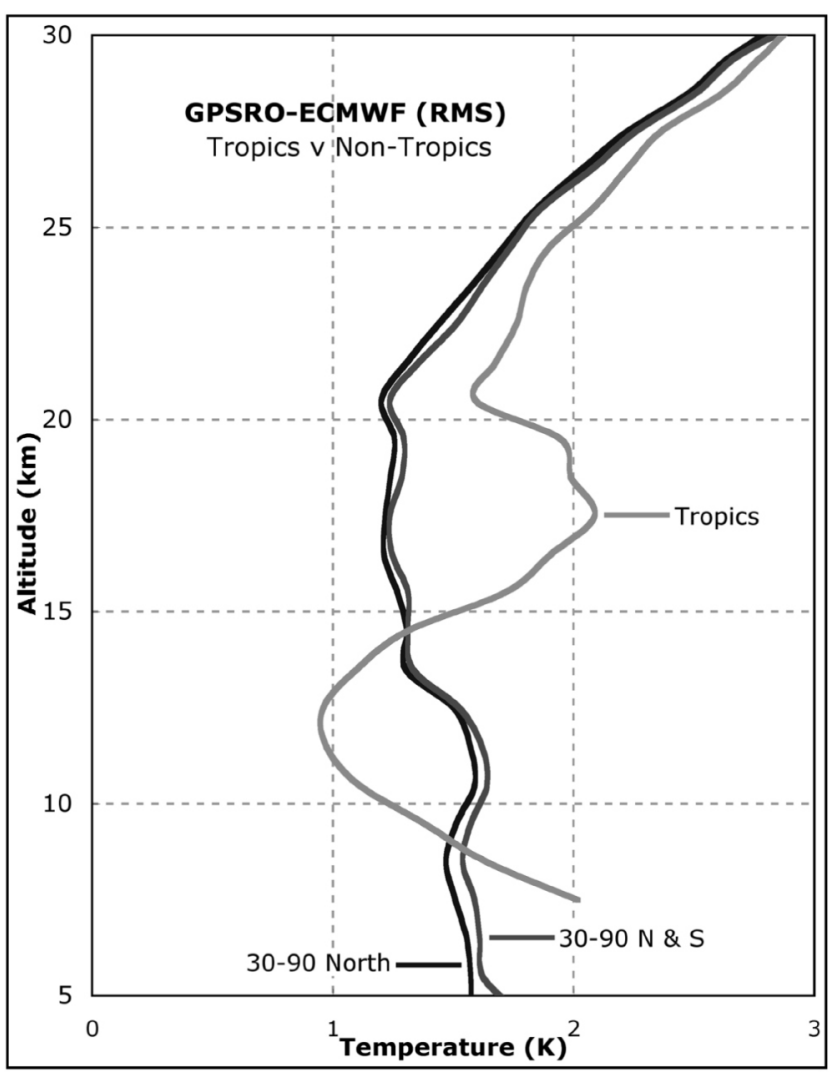

Fig. 12. Plots of the GPS-ECMWF RMS deviation for the tropics and the non-tropics, at $1 \mathrm{~km}$ resolution, illustrating the high variability of ECMWF near the often sharp tropical tropopause. 

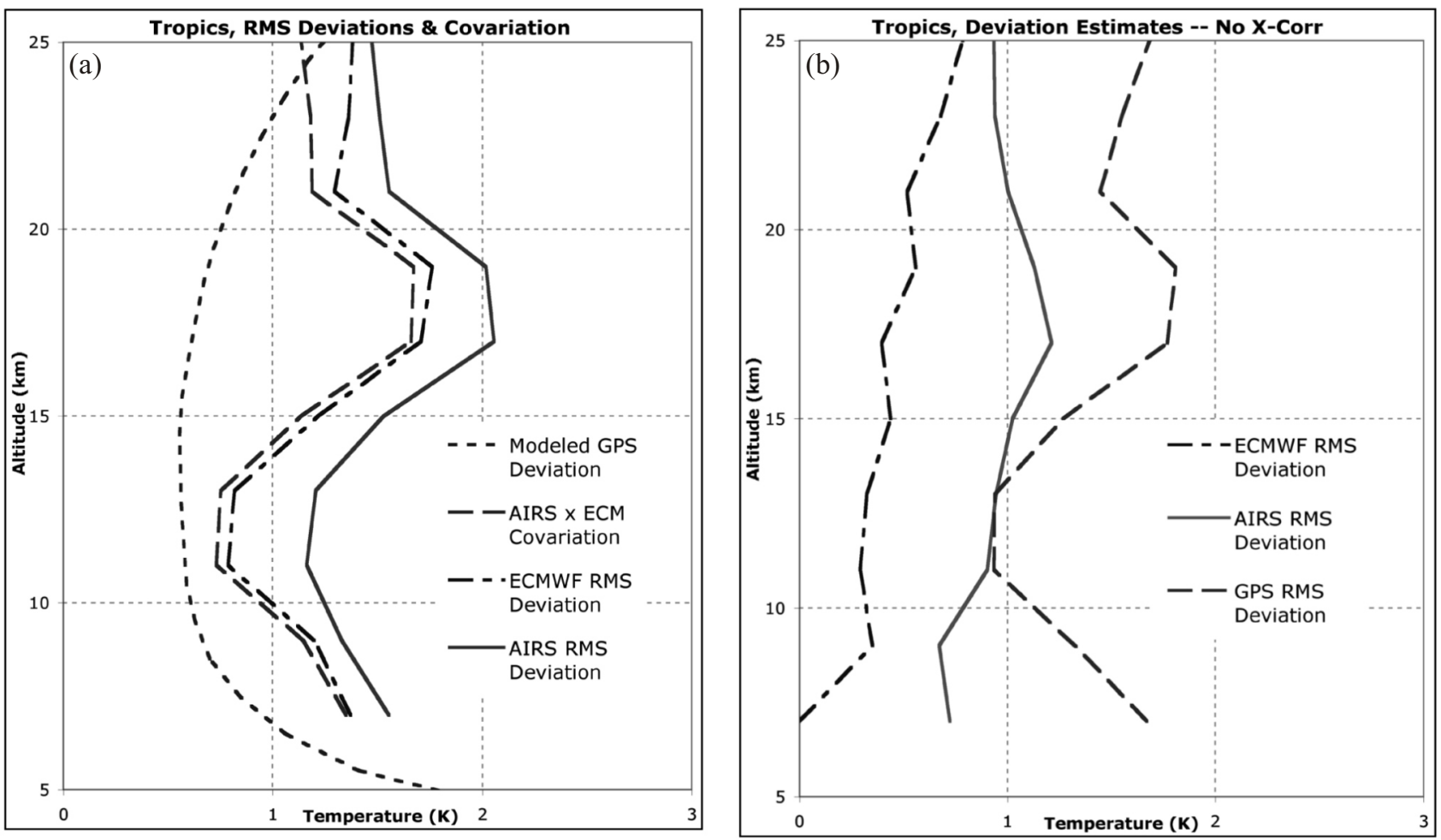

Fig. 13. Estimated AIRS and ECMWF RMS temperature deviations and the AIRS-ECMWF covariation, assuming the modeled GPS deviation (left), and the estimated RMS deviations assuming no correlation between AIRS and ECMWF profiles.

tradicts the established attribution to model deficiencies and requires a $1.8 \mathrm{~K}$ GPS-GPS covariation around the tropopause, hitherto unobserved, for which there is no known explanation. Once again the ECMWF deviation is improbably low, falling ultimately to zero, and lacks a peak at the tropopause where we expect one to occur. The results in Fig. 13b are at best implausible while those in Fig. 13a are consistent with the known or expected properties of GPSRO and ECMWF. What is surprising is the apparent high degree of correlation between AIRS and ECMWF profiles in the tropics.

\section{SUPPORTING ANALYSIS II: NORTH vs. SOUTH BIASES}

ECMWF analysis accuracy varies geographically owing to the irregular distribution of RAOBs, its most influential input. At Mid North latitudes where RAOBs abound, the model is most reliable; in the Far North and around the Southern Ocean and Antarctica, where RAOBs are scarce, the model degrades. We see this in Fig. 14 which compares the mean difference between several thousand GPS profiles and the ECMWF model for all of 2003, once again at $1 \mathrm{~km}$ resolution, in four latitude bands: Mid North $\left(30-60^{\circ} \mathrm{N}\right)$; Mid South $\left(30-60^{\circ} \mathrm{S}\right)$; Far North $\left(60-90^{\circ} \mathrm{N}\right)$; and Far South $\left(60-90^{\circ} \mathrm{S}\right)$. In the Far South, with just a few RAOBs to draw on, model deficiencies surface most vividly in the form of a highly regular vertical wave structure.

In the Mid North, with a high concentration of RAOBs, the wave structure is nearly absent. In the Mid South, which is geographically symmetric to the Mid North but which covers mostly ocean and where RAOBs are relatively sparse, the wave structure appears at an intermediate level. In the Far North, where the RAOB coverage is also intermediate to that of the Mid North and Far South, the wave pattern also emerges at an intermediate level. We see also that there is a net warm bias of a few tenths of a Kelvin in the mid latitudes not evident at high latitudes.

The known dependence of ECMWF performance on the density of available RAOB data, and the fact that GPSRO performs essentially equally at these altitudes in all four regions, indicate that the wave structure emerging outside the Mid North arises in the model. (We note that the model is not without significant inputs in the Far South. It ingests large quantities of AMSU and other satellite data, which reach their highest concentrations near the poles. That the model maintains this pronounced bias pattern in the Far South despite an abundance of satellite data says a good deal about both the value of the RAOBs and the limitations of satellite radiometry absent a solid reference.)

Figure 15 shows the GPS-ECMWF mean differences for the same four regions as Fig. 14, but restricted to profiles used in the 3-way match-ups, after smoothing to a $2 \mathrm{~km}$ re- 

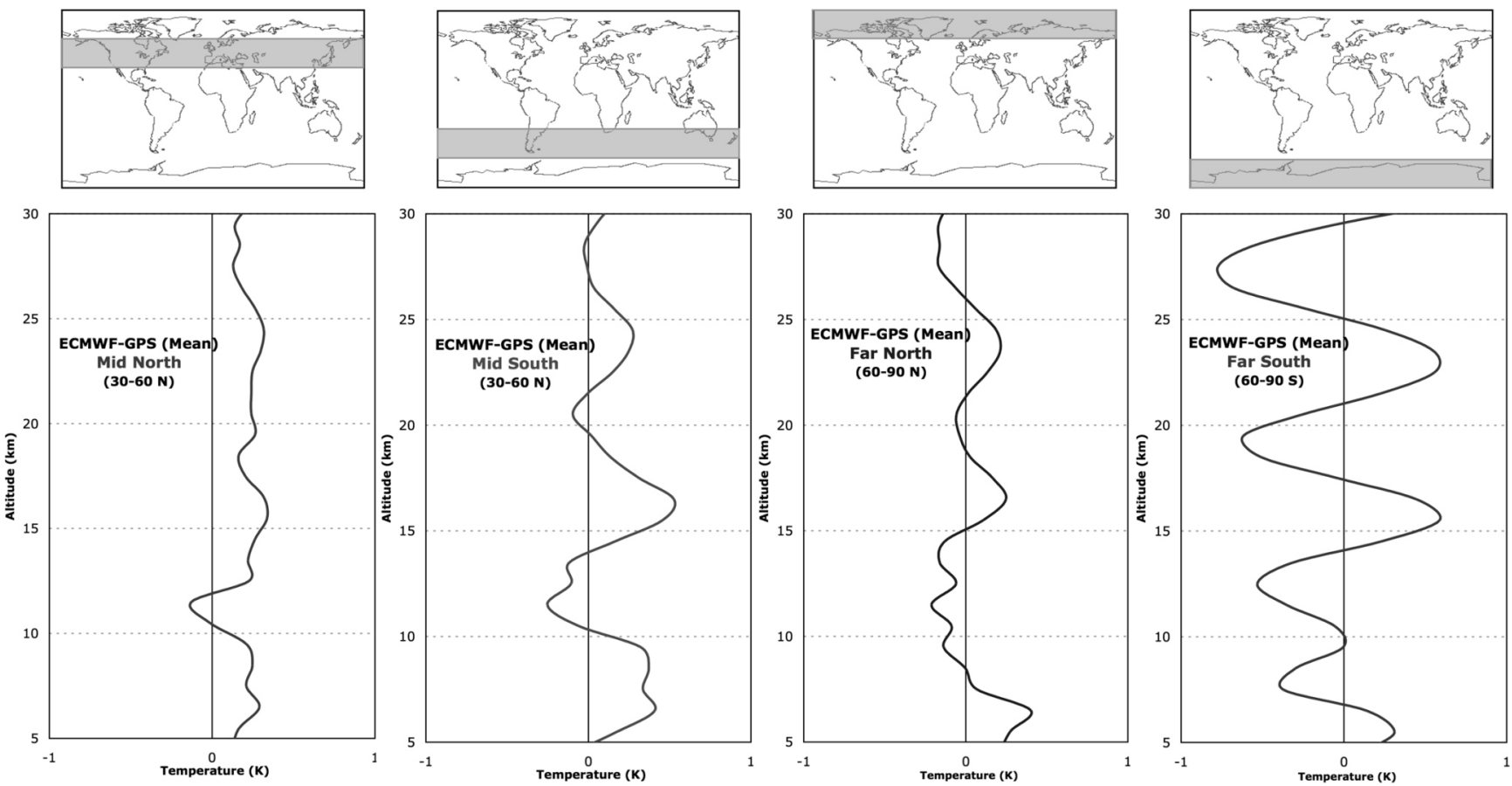

Fig. 14. Plots of the GPS-ECMWF mean offsets for four non-tropical bands, at $1 \mathrm{~km}$ resolution, illustrating the pronounced ECMWF bias pattern where RAOB inputs are limited.
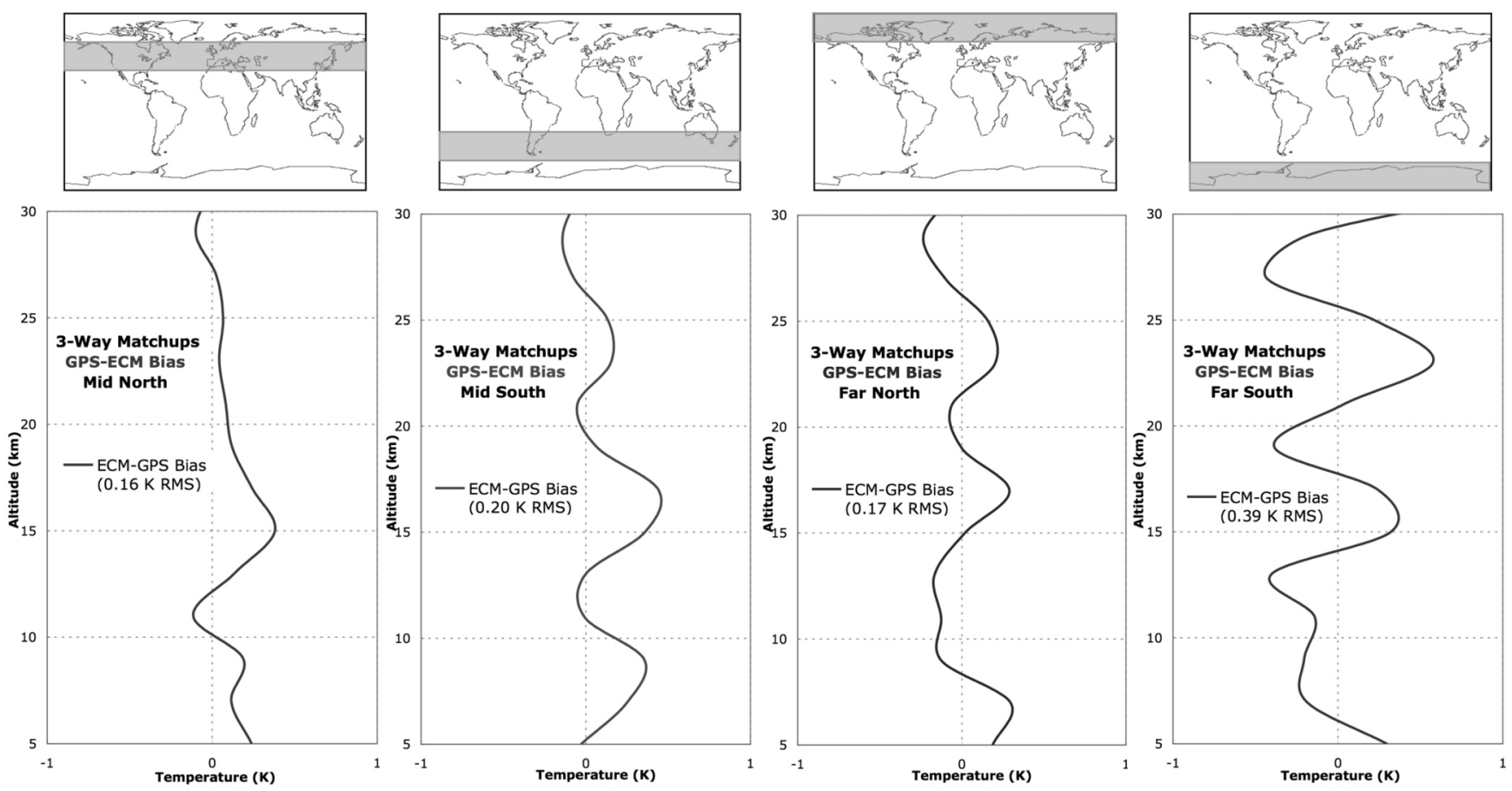

Fig. 15. Plots of the same GPS-ECMWF mean offsets as Fig. 14, but restricted to the GPS profiles having AIRS matchups and smoothed to 2 km vertical resolution.

solution. The patterns are much the same as seen in Fig. 14, although the smoothing removes vertical detail and reduces the amplitude of the Far South excursions.

Returning to our match-ups, Fig. 16 shows the same mean differences as Fig. 15 (this time drawn with line segments rather than a smooth curve) together with the GPSAIRS mean differences. In each region the GPS-AIRS mean offset pattern mimics the GPS-ECMWF pattern, par- 

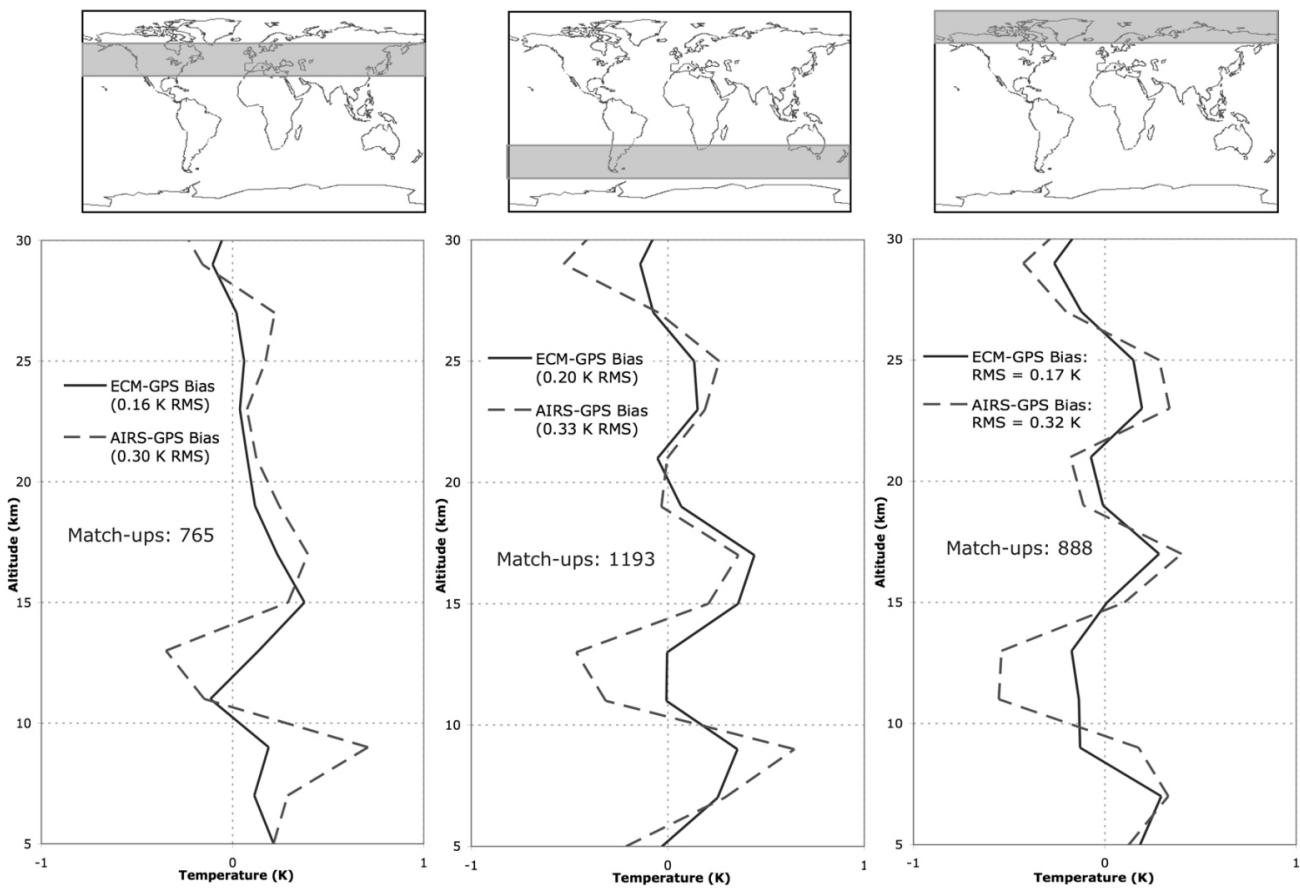
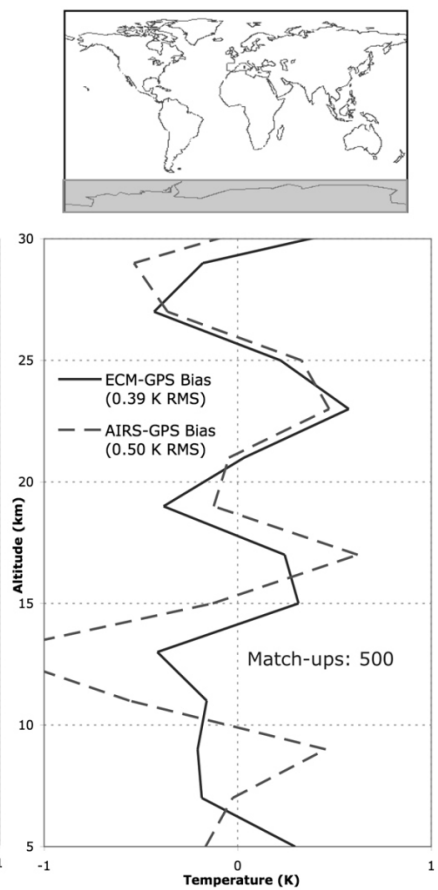

Fig. 16. Plots of the same GPS-ECMWF mean offsets as Fig. 15, along with the corresponding GPS-AIRS mean offsets.

ticularly in the appearance of a clear wave structure in the high latitudes and Mid South, virtually in phase with the ECMWF pattern. While one explanation could be that GPS is introducing common signatures, in this case we know with near certainty that (a) GPS performance above $5 \mathrm{~km}$ is essentially the same in all four regions and (b) the GPSECMWF wave structure outside the Mid North arises in the ECMWF model. We also have considerable evidence that the GPS temperature bias is below $0.1 \mathrm{~K}$ throughout this altitude range, even when smoothed to only $1 \mathrm{~km}$. The similar patterns in the GPS-ECMWF and GPS-AIRS biases indicate that the mean offsets in the latter arise mainly in the AIRS measurements, and suggest an AIRS-ECMWF correlation consistent with that inferred from RMS deviations. We see also that the bias excursions for AIRS tend to be greater than for ECMWF, with a few exceptions.

It should be noted that there is an asymmetry in the yield of match-ups between north and south: 56\% more in the Mid South than in the Mid North and 78\% more in the Far North than in the Far South. This has to do with the success of AIRS in achieving high quality retrievals in different regions. Its greater success over water than over land gives AIRS an advantage in the Mid South; its lesser success over ice and snow gives it a disadvantage within the ice fields of Antarctica.

\section{SUPPORTING ANALYSIS III: BIASES IN THE TROPICS}

Figure 17 shows the bias offsets with respect to GPS for the Tropics. As we saw with the RMS plots in Fig. 12, the pattern is quite distinct from the other regions, though certain features of the AIRS-ECMWF comparison remain. The AIRS bias pattern is grossly similar to the ECMWF pattern though with excursions stretched by about a factor of two. The warm biases for both AIRS and ECMWF at $14-17 \mathrm{~km}$ are expected if they are both smoothing down the sharp cold

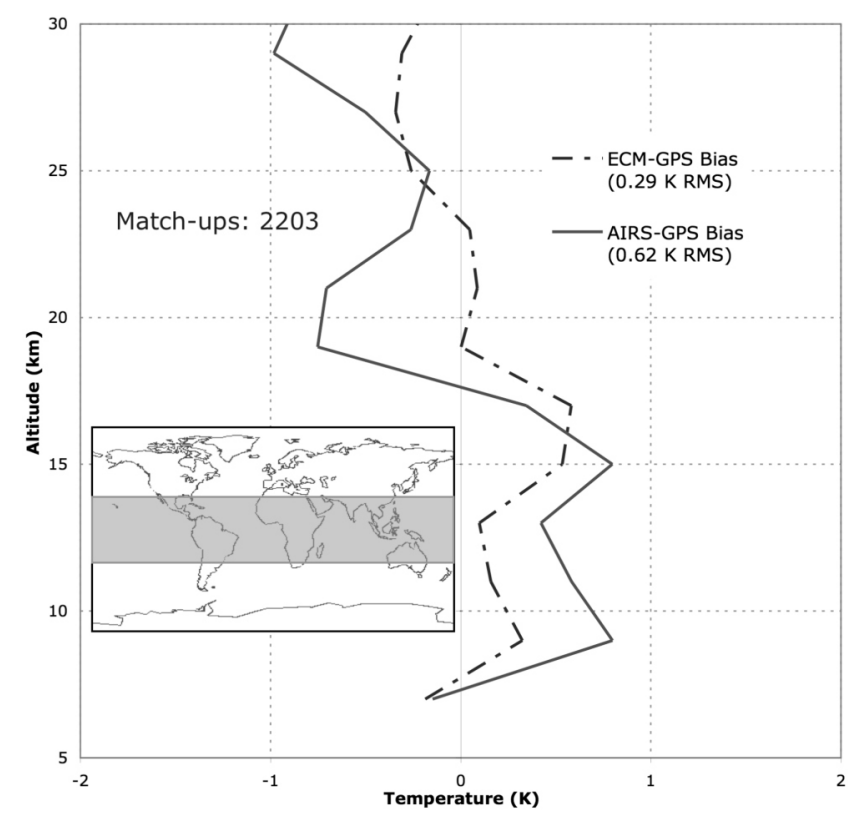

Fig. 17. Plots of GPS-ECMWF and GPS-AIRS mean offsets for 2200 match-ups in the tropics. 
peak of the tropical tropopause. This is somewhat at odds with the other regions where ECMWF is said to have a cold bias at the tropopause, though we see this only in the Far North and Far South.

For a more ready comparison, Figs. 18a - e show the bias and deviation statistics for each region, side-by-side on a common scale. Several features stand out.

1. ECMWF and (more markedly) AIRS variances peak around the tropopause, probably because the often steep gradients, positive and negative, cause them to both overshoot and undershoot in different circumstances (Fig. 11).

2. AIRS shows a consistently cold bias at or somewhat above the variance peak at the tropopause in all regions.

3. The AIRS variance is uniformly greater than the ECMWF variance in all regions.

4. The AIRS bias excursions are generally greater than the ECMWF excursions.

5. There is substantial AIRS-ECMWF covariation in all regions, greatest in the Tropics.

There is now substantial evidence that the net bias of GPSRO temperature profiles is less than $0.1 \mathrm{~K}$ between 8 and $30 \mathrm{~km}$ altitude ( $5-30 \mathrm{~km}$ outside the tropics). Indeed, that bias may be less than $0.05 \mathrm{~K}$ (Shu-peng Ho, personal communication). If that is true we can attribute virtually the entire bias excursion patterns seen in the pairwise comparisons with GPSRO to the AIRS and ECMWF components. We can then RSS together the bias and deviation estimates for each technique to yield estimates of their total error. The results are shown in Figs. 19a - f, for each region, and for the combined Far North and Far South ("Poles"). Since the bias excursions tend to be below $0.5 \mathrm{~K}$ and the individual deviations near $1 \mathrm{~K}$ or above for AIRS and ECMWF, the total error curves do not differ markedly from the deviation curves. Consequently the curves are quite similar for corresponding regions in the north and south, though the error for AIRS in the Far South is somewhat higher than in the Far North, probably because of the effects of ice and snow. In general both AIRS and ECMWF show the greatest errors around the tropopause. Figures $19 \mathrm{e}$ and $\mathrm{f}$ show clearly the different tropopause heights in the Tropics compared with the Poles.

\section{SUMMARY AND CONCLUSIONS}

As we have emphasized, one's interpretation of the RMS deviation results for AIRS and ECMWF depends on what one believes about the performance of GPSRO. We have employed a model of single-profile GPSRO temperature precision based on the best information currently available; indeed, recent unpublished results from UCAR indicate that actual GPSRO temperature error may be lower than represented in our model (Shu-peng Ho, personal communication). Application of that model reveals a signifi- cant correlation between AIRS and ECMWF temperature profiles that results in error cancellation in AIRS-ECMWF differences. If that is true, errors for individual AIRS and ECMWF profiles inferred from such differences will tend to be optimistic.

Companion studies of AIRS-GPS and ECMWF-GPS biases, which do not depend on any assumption regarding GPSRO measurement precision, tend to support the conclusion of a correlation between AIRS and ECMWF profiles. Distinctive vertical wave structures in the ECMWF-GPS bias profiles that vary by region can be definitively ascribed to deficiencies in the model resulting from limited RAOB data, a critical input that provides an absolute reference. Where RAOBs are plentiful (Mid North), the wave pattern is virtually absent; where they are fewest (Far South), it is most prominent. AIRS-GPS biases tend to show the same variation as ECMWF-GPS biases by region. While the AIRSGPS excursions are systematically greater than those for ECMWF-GPS, the wave patterns for AIRS and ECMWF are remarkably similar in each region, almost exactly in phase, with the greatest amplitude in the Far South and the smallest in the Mid North. Since the ECMWF-GPS bias patterns arise in the ECMWF model, it follows that the similar AIRS-GPS bias patterns arise largely in the AIRS retrievals.

We note that these bias excursions, while quite distinct and highly repeatable from month to month, tend to be at or below $0.5 \mathrm{~K}$ and are thus compatible with an overall error at the $1 \mathrm{~K}$ level. If we assume that our error model for GPSRO is accurate, we see that outside the tropics the ECMWF temperature error ranges between $0.6 \mathrm{~K}$ in the Far North above $20 \mathrm{~km}$ to $1.6 \mathrm{~K}$ in the Mid South near the tropopause. The AIRS temperature error ranges between 0.87 and $2.0 \mathrm{~K}$, hitting both extremes in the Far North. In the tropics, ECMWF error ranges between $0.8 \mathrm{~K}$ below the tropopause to $1.8 \mathrm{~K}$ at the tropopause, passing from one extreme to the other in the span of $4 \mathrm{~km}$. AIRS shows a very similar pattern, with larger overall errors ranging from 1.3 to $2.2 \mathrm{~K}$. The source of the apparent correlation between AIRS and ECMWF temperature errors is not well understood, but may derive in part from the AIRS retrieval initialization step, which depends upon regression coefficients trained on the ECMWF model analysis for two days in August 2002.

We note that since 2003, significant changes have been made in the ECMWF analyses, and a new AIRS "Version 5" retrieval process has been introduced. The ECMWF changes include assimilation of AIRS radiances (beginning mid 2004), increased number of vertical levels (January 2006), and assimilation of GPSRO profiles (December 2006). These changes will undoubtedly have significant effects on the comparison statistics. We are now in the process of repeating the comparisons with data acquired after each of these milestones to quantify the changing relationship among the three datasets.

Perhaps the larger message from these studies is that, 
(a)

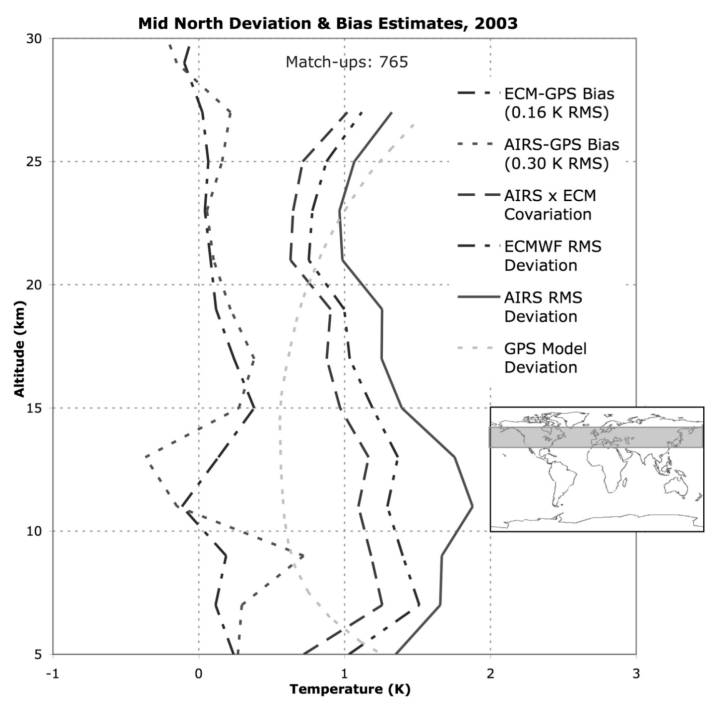

(c)

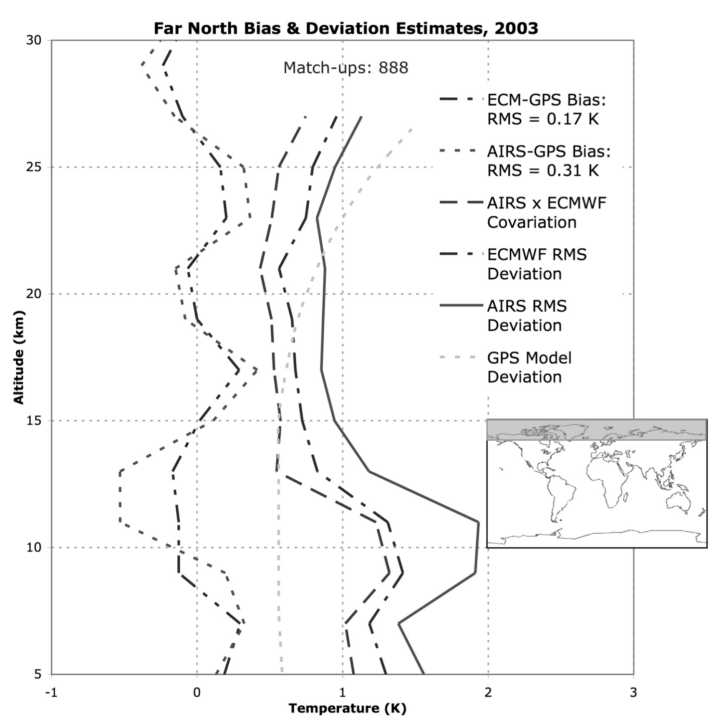

(e)

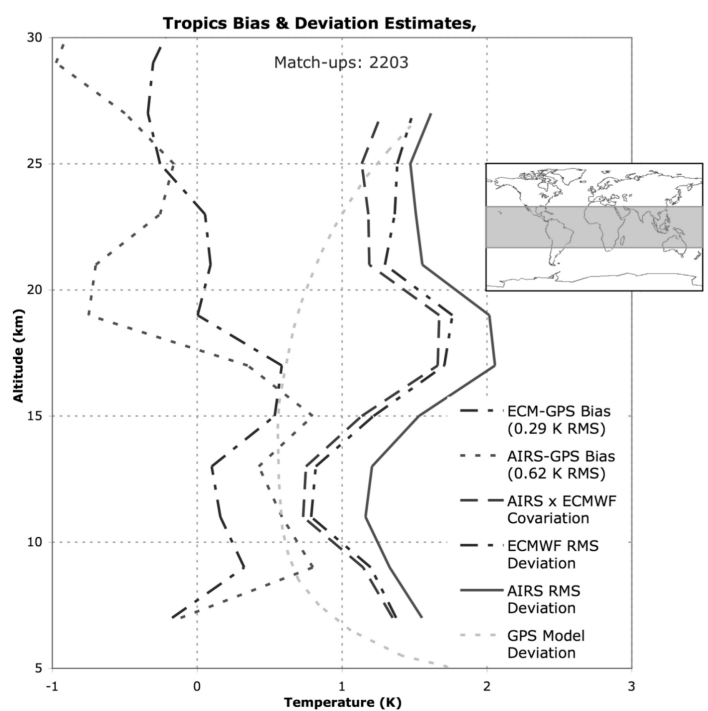

(b)

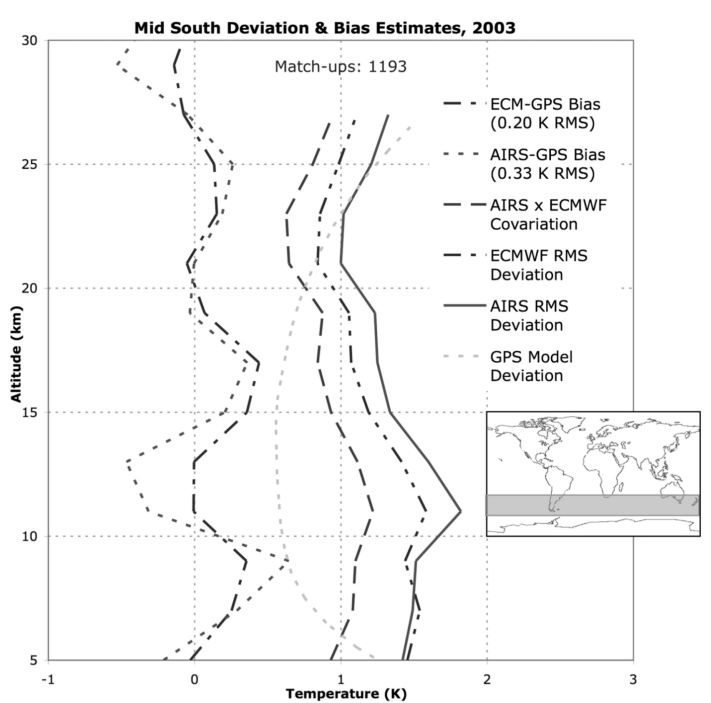

(d)

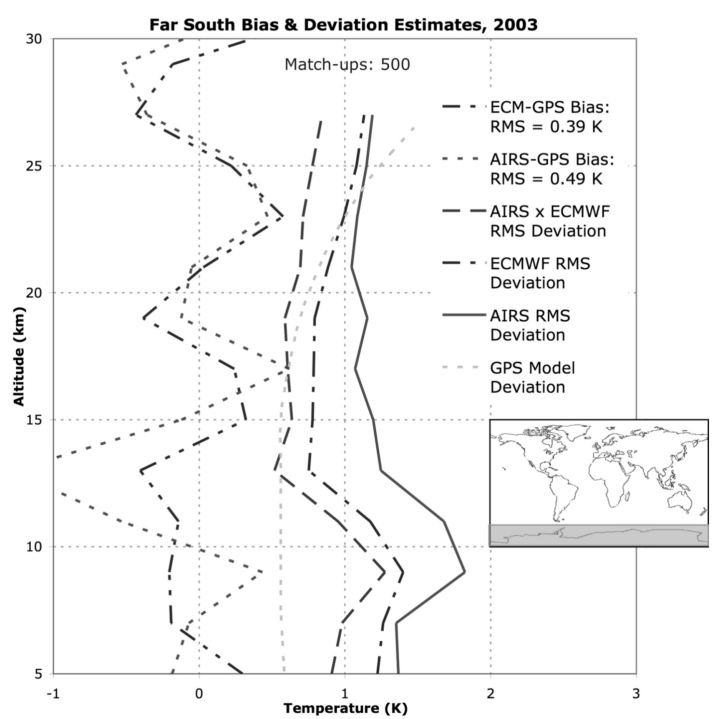

Fig. 18. Side-by-side plots of the GPS-ECMWF and GPSAIRS mean offsets for 2003 along with estimates of individual AIRS and ECMWF deviations and the AIRSECMWF covariation for all five latitude bands examined. 
(a)

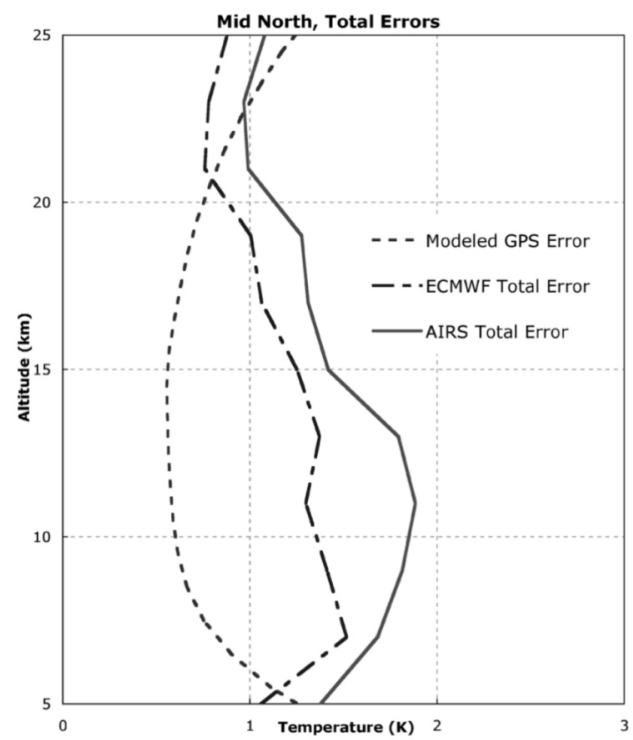

(c)

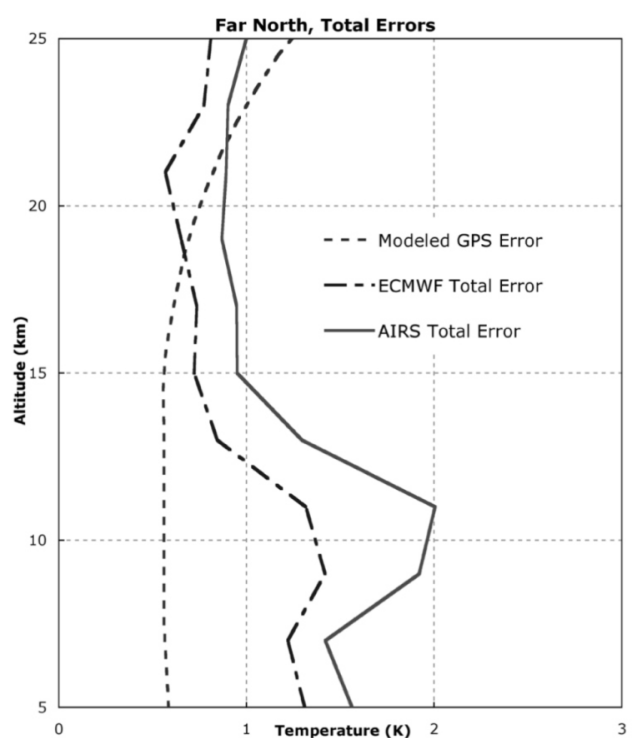

(e)

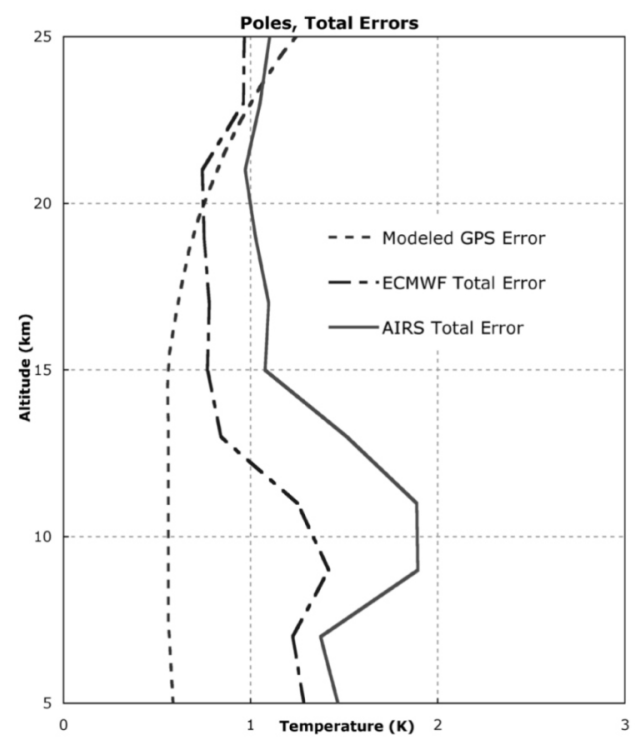

(b)

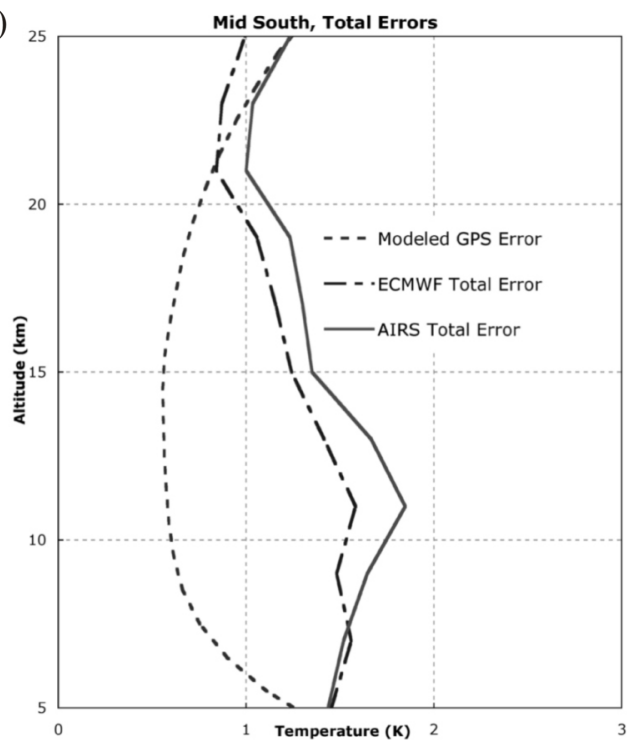

(d)

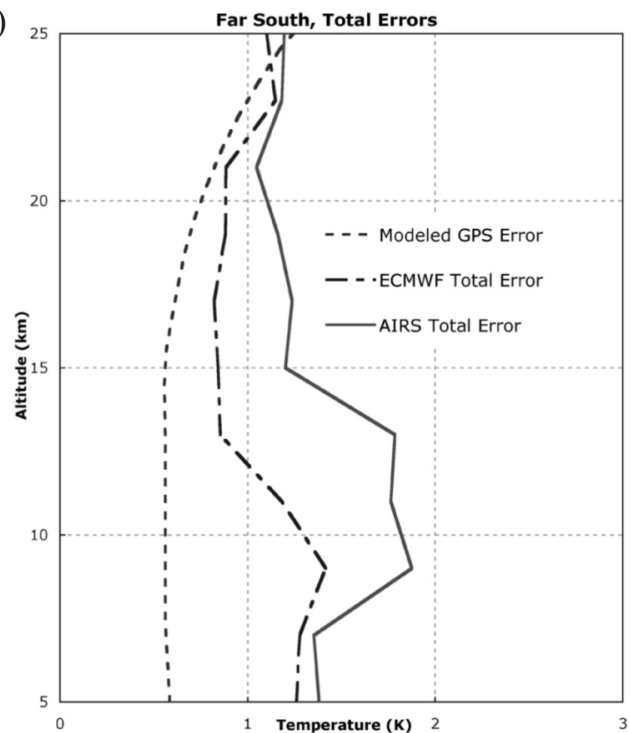

(f)

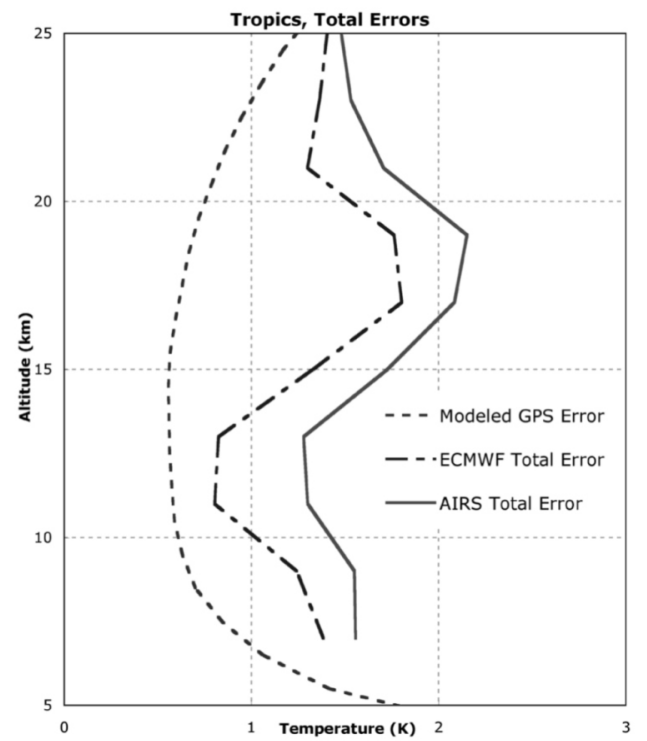

Fig. 19. Total error plots for AIRS, ECMWF, and GPSRO temperature profiles for each of the five regions and for the combined high latitudes ("Poles"). 
should the error model employed here for GPSRO temperature profiles be substantiated in future studies, then GPSRO will serve as an effective, nearly definitive means for charting the performance of spaceborne radiometers in retrieving atmospheric temperatures.

Acknowledgement The research described in this paper was conducted at the Jet Propulsion Laboratory under contract with the National Aeronautics and Space Administration.

\section{REFERENCES}

Anthes, R., 2006: Welcome to a new era! Keynote lecture, Formosat-3/COSMIC Workshop in Taiwan, 28 November 2006.

Chahine, M. T., T. S. Pagano, H. H. Aumann, R. Atlas, C. Barnet, J. Blaisdell, L. Chen, M. Divakarla, E. J. Fetzer, M. Goldberg, C. Gautier, S. Granger, S. Hannon, F. W. Irion, R. Kakar, E. Kalnay, B. H. Lambrigtsen, S.-Y. Lee, J. L. Marshall, W. W. Mcmillan, L. Mcmillin, E. T. Olsen, H. Revercomb, P. Rosenkranz, W. L. Smith, D. Staelin, L. L. Strow, J. Susskind, D. Tobin, W. Wolf, and L. Zhou, 2006: AIRS improving weather forecasting and providing new data on greenhouse gasses. Bull. Amer. Meteor. Soc., 87, 911-926, doi: 10.1175/BAMS-87-7-911. [Link]

Divakarla, M. G., C. D. Barnet, M. D. Goldberg, L. M. McMillin, E. Maddy, W. Wolf, L. Zhou, and X. Liu, 2006: Validation of Atmospheric Infrared Sounder temperature and water vapor retrievals with matched radiosonde measurements and forecasts. J. Geophys. Res., 111, D09S15, doi: 10.1029/2005JD006116. [Link]

Fetzer, E. J., A. Eldering, E. F. Fishbein, T. Hearty, W. F. Irion, and B. Kahn, 2005: AIRS/AMSU/HSB validation report for version 4.0 data release. Jet Propulsion Laboratory,
D-31448, 8 March 2005, 61 pp.

Hajj, G. A., C. O. Ao, B. A. Iijima, D. Kuang, E. R. Kursinski, A. J. Mannucci, T. K. Meehan, L. J. Romans, M. D. Juarez, and T. P. Yunck, 2004: CHAMP and SAC-C atmospheric occultation results and intercomparisons. J. Geophys. Res., 109, D06109, doi: 10.1029/2003JD003909. [Link]

Healy, S. B. and J. N. Thépaut, 2006: Assimilation experiments with CHAMP GPS radio occultation measurements. Quart. J. Roy. Meteor. Soc., 132, 605-623, doi: 10.1256/qj.04.182. [Link]

Ho, S. P., M. Goldberg, Y. H. Kuo, C. Z. Zou, and W. Schreiner, 2009: Calibration of temperature in the lower stratosphere from microwave measurements using COSMIC radio occultation data: Preliminary results. Terr. Atmos. Ocean. Sci., 20, 87-100, doi: 10.3319/TAO.2007.12.06.01(F3C). [Link]

Kuo, Y. H., T. K. Wee, S. Sokolovskiy, C. Rocken, W. Schreiner, D. Hunt, and R. A. Anthes, 2004: Inversion and error estimation of GPS radio occultation data. J. Meteorol. Soc. Jpn., 82, 507-531, 0026-1165, ISI: 000221414100023, doi: 10.2151/jmsj.2004.507. [Link]

Kursinski, E. R., G. A. Hajj, J. T. Schofield, R. Linfield, and K. R. Hardy, 1997: Observing Earth's atmosphere with radio occultation measurements using the Global Positioning System. J. Geophys. Res., 102, 23429-23465, doi: 10.1029/ 97JD01569. [Link]

Leroy, S. S., 1997: Measurement of geopotential heights by GPS radio occultation. J. Geophys. Res., 102, 6971-6986, doi: 10.1029/96JD03083. [Link]

Rocken, C., R. Anthes, M. Exner, D. Hunt, S. Sokolovskiy, R. Ware, M. Gorbunov, W. Schreiner, D. Feng, B. Herman, Y. H. Kuo, and X. Zou, 1997: Verification of GPS/MET data in the neutral atmosphere. J. Geophys. Res., 102, 2984929866, doi: 10.1029/97JD02400. [Link] 